\title{
Séminaire sur les Pensées de Pascal
}

Compte-rendu général 2000-2001

\section{Dominique Descotes}

\section{(2) OpenEdition}

\section{Journals}

Édition électronique

URL : http://journals.openedition.org/ccibp/535

DOI : $10.4000 /$ ccibp.535

ISSN : 2493-7460

Éditeur

Centre international Blaise Pascal

\section{Édition imprimée}

Date de publication : 21 novembre 2002

Pagination : 26-46

ISBN : 2-84516-215-4

ISSN : 0249-6674

Référence électronique

Dominique Descotes, «Séminaire sur les Pensées de Pascal », Courrier du Centre international Blaise Pascal [En ligne], 24 | 2002, mis en ligne le 02 décembre 2015, consulté le 20 avril 2019. URL : http:// journals.openedition.org/ccibp/535

Ce document a été généré automatiquement le 20 avril 2019.

Centre international Blaise Pascal 


\title{
Séminaire sur les Pensées de Pascal
}

\author{
Compte-rendu général 2000-2001
}

\author{
Dominique Descotes
}

1 À l'initiative de Laurent Thirouin, Antony McKenna et Dominique Descotes, le CIBP organise un séminaire consacré aux Pensées.

\section{Projet du séminaire}

2 Son objet est un examen méthodique et ordonné des fragments des Pensées, en vue de constituer un état des sources et des principaux commentaires, et de faire le point sur les interprétations majeures dont les textes ont fait l'objet. Le séminaire procédera à l'examen par ordre des fragments des Pensées, sans chercher à passer rapidement aux plus connus ou aux plus étoffés. Le travail sur chaque fragment suivra autant que possible le modèle suivant :

1. Recensement des problèmes posés par l'établissement du texte et des études correspondantes,

2. Recensement des sources,

3. Élucidations terminologiques,

4. Recensement des interprétations majeures des fragments,

5. Recensement des renvois entre les fragments des Pensées.

Une bibliographie critique aussi complète que possible sera réunie pour chaque fragment, qui retiendra les études qui ont apporté une avancée marquante ou proposé une interprétation qu'il est impossible d'ignorer ; elle sera étoffée progressivement, selon les apports des participants. Pour chaque dossier (ou liasse) une bibliographie d'ensemble sera établie. Les apports des participants seront recueillis et mis en forme, en vue de la constitution d'une base de données informatique qui sera mise à la disposition des pascalisants. Ils serviront aussi à préciser la politique d'acquisition documentaire du CIBP en fonction des besoins qui apparaîtront. 
4 L'édition de référence est l'édition de Philippe Sellier mise à jour par Gérard Ferreyrolles, dans le Livre de Poche, qui fournit la correspondance directe avec la numérotation Lafuma. Le travail de chaque séance sera préparé par un examen de certaines éditions remarquables, confié à un participant permanent. Seront systématiquement consultées les éditions suivantes : l'édition de Port-Royal, Havet, Brunschvicg, Tourneur, Lafuma Luxembourg, Le Guern, et à la demande de certains participants, l'édition Martineau. Les résultats des travaux de Pol Ernst et de Yoichi Maeda seront systématiquement rapportés: indication de la strate avec les données de datation correspondantes, voisinage des fragments sur les feuillets originaux, couches de texte. Est souhaitée aussi une enquête suivie sur les rapprochements avec le texte et les notes de la Bible de Saci et les écrits des membres du groupe de Port-Royal.

5 Pour des raisons de mise au point de la méthode de travail, il a été décidé de commencer par la Table des matières et par la liasse Ordre.

Le séminaire s'adresse aux jeunes chercheurs qui poursuivent actuellement la rédaction d'une thèse tout autant qu'aux pascalisants dont les publications sont déjà connues. Les séances se tiendront, alternativement à Clermont-Ferrand et à Lyon, au rythme d'une tous les deux mois environ. Une participation suivie aux séances est indispensable.

7 Les correspondants du CIBP reçoivent le programme de chaque séance de ce séminaire sur demande adressée à Dominique Descotes, Centre International Blaise Pascal, Bibliothèque, 1 boulevard Lafayette, 63000 Clermont-Ferrand. Les personnes qui s'associent au séminaire sont invitées à communiquer leurs commentaires et leurs compléments au CIBP. Ces apports seront examinés et discutés à la séance suivante, en vue de corriger et de compléter le procès-verbal. La présente publication doit permettre de recueillir de nouvelles contributions, qui seront publiées après examen en séance. Les correspondants peuvent recevoir les comptes rendus des séances par courrier électronique.

Renseignements : Dominique.DESCOTES@univ-bpclermont.fr

9 Par $O C$, nous désignons les CEuvres complètes de Pascal éditées par Jean Mesnard, Bibliothèque européenne, Desclée de Brouwer, 4 vol. parus.

Par GEF, nous désignons les CEuvres complètes de Pascal dans l'édition des Grands Écrivains de la France, par Brunschvicg, Boutroux et Gazier.

\section{La « table des matières »}

11 Photographie du texte, voir Les Pensées de Pascal ont trois cents ans, Planche II (Première table de la Première Copie) et Planche III (Seconde Copie).

12 Sur la situation de la table dans les deux copies, voir MESNARD Jean, « Aux origines de l'édition des Pensées: les deux Copies", p. 8; présence de deux exemplaires dans la première Copie en tête et p. 189 du manuscrit) ; présence unique dans la seconde Copie (en tête de la Copie) : p. 11.

13 Sur les difficultés offertes par certains titres (Raisons des effets, Opinions du peuple saines, Prophéties),

- MESNARD Jean, « Aux origines de l'édition des Pensées : les deux Copies », p. 26.

- HIROTA Masayoshi, « Sur les deux Copies des Pensées de Pascal » (en japonais), in Gengo bunka , n 7, Université Hitosyubashi, mars 1971, p. 85-95. 


\section{Éléments de bibliographie}

- BARnEs Annie, «La table des titres de la copie des Pensées est-elle de Pascal ? », French studies, 1953, p. 140-146.

- CARRAud Vincent, Pascal et la philosophie, p. 93-102.

- COLE John R., « Pascal's plans for the Apology: two successive conceptions in the two-column table ", French studies, vol. L, n 1, january 1996, p. 15-34.

- hirota Masayoshi, «Sur les deux Copies des Pensées de Pascal » (en japonais), in Gengo bunka, n 7, Université Hitosyubashi, mars 1971, p. 85-95.

- Mesnard Jean, «Aux origines de l'édition des Pensées: les deux Copies ", in Les Pensées de Pascal ont trois cents ans, De Bussac, Clermont-Ferrand, 1971, p. 1-29. Voir p. 25 sur la nature de la table de la Première Copie. Comparaison de la situation des tables dans les deux Copies : p. 26.

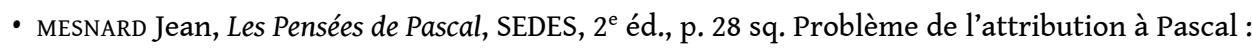
p. 28 sq. Sa nature de copie figurée : p. 30. La table des matières comme pensée : p. 30.

- SEllier Philippe, Port-Royal et la littérature, I, Pascal, p. 51 sq. Sur la nature du fragment 1 (éd. Sellier). Critique de l'interprétation du fragment comme sommaire de l'œuvre projetée selon la succession linéaire des chapitres. Simple récapitulatif des dossiers, dans l'état où les proches de Pascal les ont découverts : p. 51-52. Après le classement de juin 1658, Pascal n'a travaillé que sur les dossiers de la colonne de droite : Commencement, Excellence, La nature est corrompue, Loi figurative, Perpétuité, Preuves de Moïse, Prophéties; p. 78-79. Inutilité de considérer le texte comme une copie figurée : p. 79.

- Une référence utile : MESNARD Jean, « Pourquoi les Pensées de Pascal se présentent-elles sous forme de fragments? ", Papers on French Seventeenth Century Literature, vol. X, n 19, 1983, p. 635-649.

14 La discussion porte principalement sur l'importance de l'enjeu rhétorique enveloppé dans les interprétations existantes de ce texte, soit comme fragment de Pascal, soit comme texte faisant écran devant son objectif; comme table de dossiers, de thèmes ou de chapitres. On s'accorde à éviter de parler de plan ou de sommaire. On se contente (prudemment) de parler d'état des lieux de 1658.

\section{Recherches d'éclaircissements}

15 On recherche des éclaircissements et des références bibliographiques sur les points suivants :

1. Existe-t-il une étude sur les titres des liasses dans le manuscrit original ?

2. Existe-t-il des études sur l'abréviation des titres dans la liste?

3. Le mot-titre Rabbinage: on recherche des éclaircissements sémantiques. Le mot n'apparaît pas dans les fragments de la liasse dont il est le titre (contrairement à Vanité ou Raison des effets par exemple). En existe-t-il des occurrences en dehors des Pensées? Le Dictionnaire de l'Académie fournit les indications suivantes : « RABBINAGE. s. m. Ce nom n'a d'usage que pour signifier l'étude qu'on fait des livres des Rabbins. C'est un homme qui passe sa vie dans le rabbinage. Il ne se dit guère que par mépris. » Rabbinage se trouve, apparemment sans nuance méprisante, dans MERSENNE, Correspondance, IV, p. 35, lettre de Mersenne à Rivet du 8 février 1634 : le P. Philippeau « fait état de ne rien imprimer de tout ce qui se trouve dans les autres auteurs et il est tout rempli de passages tirés de Misne Thora, des manuscrits Grecs et Arabes, qu'il tourne en latin après les avoir cités; il ponctue aussi le rabbinage pour le 
rendre plus aisé ». Voir p. 71, lettre à Rivet du 12 mars 1634, sur le P. Philippeau et son livre sur Osée : « il y aura grande quantité de Rabbinage, de Grec et d'Arabe ».

\section{La liasse « Ordre »}

\section{Bibliographie d'ensemble de Ordre}

On s'entend pour ne pas envisager ici l'ordre comme concept logique, sur quoi la référence de base est PRIGENT Jean, "La conception pascalienne de l'ordre", Ordre, désordre, lumière, Paris, 1952, p. 190-209. Ordre s'entend ici seulement comme le titre de l'ensemble des fragments Sel. 37-46.

- ERNST Pol, Les Pensées de Pascal. Géologie et stratigraphie, Voltaire Foundation Universitas, 1996, Oxford, $480 \mathrm{p}$.

- MAeda Yoichi, Commentaire des Pensées, I.

- SELLIER Philippe, Port-Royal et la littérature, I, Pascal, p. 55 sq.

- soelberg Nils, «La dialectique de Pascal. De la conférence de Port-Royal à la démarche apologétique », Revue romane $t$. XIII, fasc. 2, 1978, p. 229-276.

- suematsu Hisashi, « Les Pensées et le métatexte », Équinoxe, 1, automne 1987, p. 27-53.

\section{Aspects stratigraphiques des fragments de Ordre}

ERNST Pol, Les Pensées de Pascal. Géologie et stratigraphie, Voltaire Foundation Universitas, 1996, Oxford, $480 \mathrm{p}$. Voir :

- p. 290. Le fragment Sel. 37 est sur papier $\varepsilon$ feuillet à réglure.

- p. 290. Les fragments Laf. 2 à 4, Sel. 38 ; sont à la marque royale.

- p. 285 : Appartiennent à la strate FNIC

- Laf. 5, SEL. 39

- Laf. 7, Sel. 41

- Laf. 8, Sel. 42

- Laf. 9, Sel. 43

- Laf. 11, Sel. 45

Sur l'époque à laquelle Pascal a utilisé les feuilles de la strate FNIC, voir ERNST Pol, Les Pensées de Pascal. Géologie et stratigraphie, p. 241 sq.

19 Nota bene: pour citer le livre de P. Ernst, les références de l'édition Lafuma telles que l'auteur lui-même les donne sont conservées afin d'éviter les équivoques dues aux différences dans le découpage des fragments. Chacun rétablira aisément la correspondance avec l'édition Sellier.

\section{Titre}

Le titre Ordre est donné en tête du dernier fragment de la liasse, Sel. 46, de la main de Pascal.

21 Suematsu Hisashi, «Les Pensées et le métatexte », Équinoxe, 1, automne 1987, p. 27-53. Voir p. 45 : le titre d'Ordre ne se réfère pas à un sujet de l'Apologie ni à la signification qu'elle est censée produire mais traite de la composition et de la disposition de la matière : p. 45. Cette liasse est différente des autres, à l'exception peut-être de APR, dont le titre n'a pas 
de rapport avec le contenu discursif, de Rendre la religion aimable, de Commencement et de Conclusion.

\section{Fonction de la liasse Ordre}

- PRIGENT Jean, «La notion d'ordre chez Pascal», in Ordre, désordre, lumière, Vrin, Paris, p. 190-209.

- ERNST Pol, Approches pascaliennes, p. 46. Interprétation de la liasse Ordre comme « un projet d'introduction générale » qui « devait comprendre deux Lettres », la Lettre qu'on doit chercher Dieu, et la Lettre d'ôter les obstacles, suivies de "précisions relatives au dessein général de l'Apologie » (p. 46).

- gounier Henri, Blaise Pascal. Commentaires, p. 181.

- suematsu Hisashi, «Les Pensées et le métatexte », Équinoxe, 1, automne 1987, p. 27-53. Voir p. 44 sq. Insiste sur le caractère presque entièrement métatextuel de la liasse Ordre: p. 45.

On remarque la fréquence avec laquelle Pascal annonce préalablement, sinon le plan de son ouvrage, du moins la succession des arguments ou des effets à produire (Provinciale XI, Provinciale XV, Premier Écrit des curés de Paris, Traité de la prédestination, Discours sur la possibilité des commandements, Lettre à Carcavy, etc.) ; et le paradoxe qui fait qu'une liasse intitulée Ordre contient des fragments qui se caractérisent surtout par une grande diversité d'orientations.

Voir les Pensées, éd. Sellier, p. 40, n. 1. Philippe Sellier note que Ordre rassemble des notes où, lors de l'agencement des vingt-sept rubriques provisoires de l'Apologie, Pascal a indiqué les projets nouveaux qui germaient en lui.

\section{Table de concordance}

24 Cf. Les deux tables de concordance de l'édition Sellier (Classique Garnier, 1991, p. 624-634), et de l'édition Brunschvicg (Euvres, Pensées de Blaise Pascal, t. 1, Hachette, 1904, p. CCLXXVI - CCCIV).

\begin{tabular}{|c|c|c|c|c|c|}
\hline Sellier & Lafuma & Brunschvicg & & $\begin{array}{l}\text { Havet }\left(4^{\mathrm{e}} \text { éd. }\right. \\
\text { de } 1887)\left(2^{\mathrm{e}}:\right. \\
1866 \text { et } 3^{\mathrm{e}}: \\
1881)\end{array}$ & $\begin{array}{l}\text { Remarques (absence dans les éditions } \\
\text { antérieures: PR [1670], Bossut [1779], } \\
\text { Faugère [1844], Havet [1 ère éd. de 1852], } \\
\text { Molinier [1877-1879] }\end{array}$ \\
\hline S. 37 & L. 1 & B. 596 & $\begin{array}{l}\text { Ms. } \\
27\end{array}$ & H. XXV. 45 & abs. ds P.R. et ds Boss. \\
\hline S. 38 & L. 2 & B. 227 & $\begin{array}{l}\text { Ms. } \\
29\end{array}$ & H. XXV. 109 & abs. ds P.R. et ds Boss. \\
\hline S. 38 & L. 3 & B. 244 & $\begin{array}{l}\text { Ms. } \\
29\end{array}$ & H. XXV. 200 & abs. ds P.R. et ds Boss. \\
\hline S. 38 & L. 4 & B.184 & $\begin{array}{l}\text { Ms. } \\
29\end{array}$ & $\begin{array}{lll}\text { H. XXV. } 108 \\
\text { bis }\end{array}$ & abs. ds P.R. et ds Boss. \\
\hline
\end{tabular}




\begin{tabular}{|c|c|c|c|c|c|}
\hline $\begin{array}{ll}\text { S. } & 39 \\
247 & \end{array}$ & L. 5 & B. 247 & $\begin{array}{l}\text { Ms. } \\
25\end{array}$ & H. X. 9 & abs. ds P.R., Boss. et la $1^{\text {ère }}$ éd. \\
\hline $\begin{array}{ll}\text { S. } & 40 \\
60 & \end{array}$ & L. 6 & B. 60 & $\begin{array}{l}\text { Ms. } \\
25\end{array}$ & H. XXII 1 & abs. ds P.R., et Boss. \\
\hline S. 41 & L. 7 & B. 248 & $\begin{array}{l}\text { Ms. } \\
25\end{array}$ & H. X 11 & abs. ds P.R., Boss. et la $1^{\text {ère }}$ éd. \\
\hline $\begin{array}{ll}\text { S. } & 42 \\
602 & \end{array}$ & L. 8 & B. 602 & $\begin{array}{l}\text { Ms. } \\
27\end{array}$ & H. XXV. 199 & abs. ds P.R., Boss. et la $1^{\text {ère }}$ éd. \\
\hline S. 43 & L. 9 & B. 291 & $\begin{array}{l}\text { Ms. } \\
25\end{array}$ & H. XXV. 110 & abs. ds P.R., et Boss. \\
\hline S. 44 & L. 10 & B. 167 & $\begin{array}{l}\text { Ms. } \\
27\end{array}$ & H. abs. & abs. ds P.R., et Boss. \\
\hline S. 45 & L. 11 & B. 246 & $\begin{array}{l}\text { Ms. } \\
25\end{array}$ & H. X. 10 & abs. ds P.R., Boss. et la $1^{\text {ère }}$ éd. \\
\hline S. 46 & L. 12 & B. 187 & $\begin{array}{l}\text { Ms. } \\
27\end{array}$ & H. XXIV. 26 & \\
\hline
\end{tabular}

\section{Sel. 37}

Les psaumes chantés par toute la terre.

Qui rend témoignage de Mahomet? Lui-même.

Jésus-Christ veut que son témoignage ne soit rien.

La qualité de témoins fait qu'il faut qu'ils soient toujours et partout, et misérables. Il est seul. 


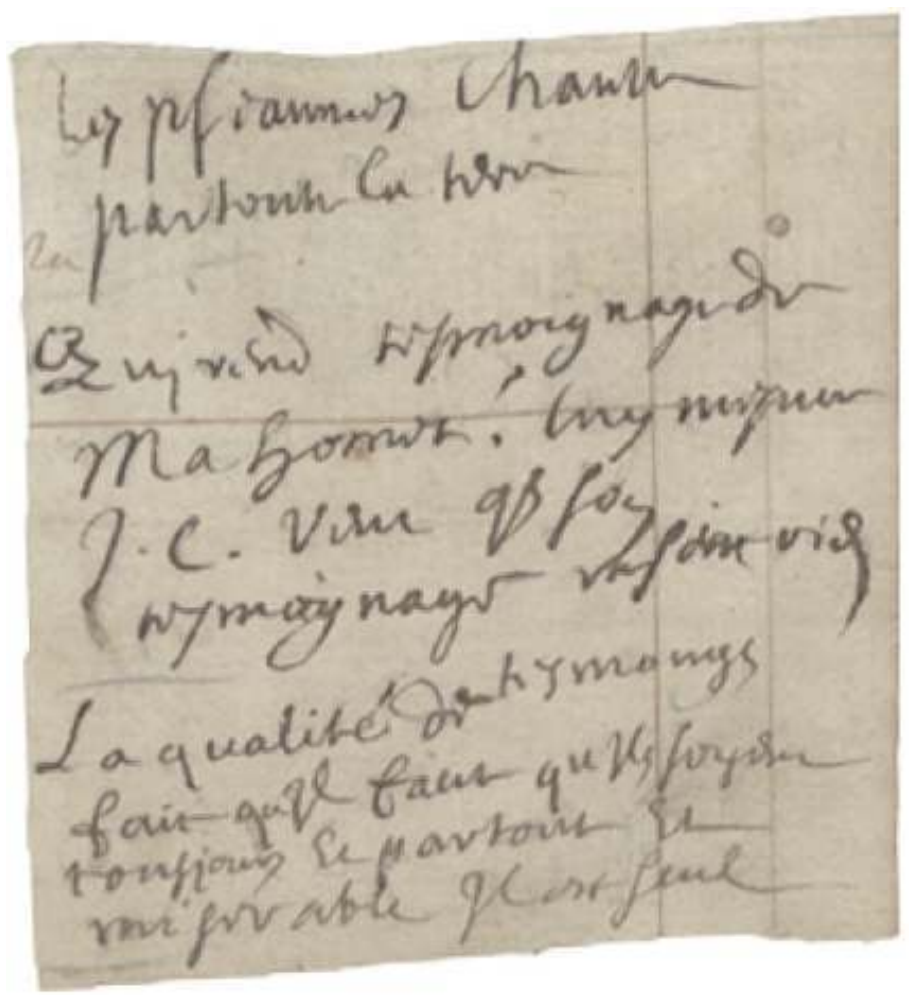

BNF

\section{Manuscrit}

- Maeda Yoichi, Commentaire des Pensées, I, p. 4, et p. 7-8; "Sur un fragment des Pensées », in Mélanges en l'honneur de Kinji Shimada, Tokyo, 1986, p. 27-32. Le texte, d'abord écrit au crayon, a été repassé à l'encre.

- ERNST Pol, Les Pensées de Pascal. Géologie et stratigraphie, Voltaire Foundation Universitas, 1996, Oxford, 480 p. Voir p. $290: \varepsilon$ feuillet à réglure. Voir la référence dans ERNST Pol, Les Pensées de Pascal. Géologie et stratigraphie, p. 290 ; mais la photographie de la reconstitution n'est pas donnée dans l'ouvrage publié par la Voltaire Foundation. Texte situé sur les vestiges d'un feuillet à réglures sur lequel sont placés plusieurs fragments : Prophéties, L 330 ; Prophéties, L 325 ; Série I, L 393 ; Série I, L 394 ;Fausseté, L 204 ; XXIV, L 624 ; Série I, L 386 ; Perpétuité, L 279 ; Ordre, L 1. 
Texte situé sur les vestiges d'un feuillet à réglures sur lequel sont placés plusieurs fragments
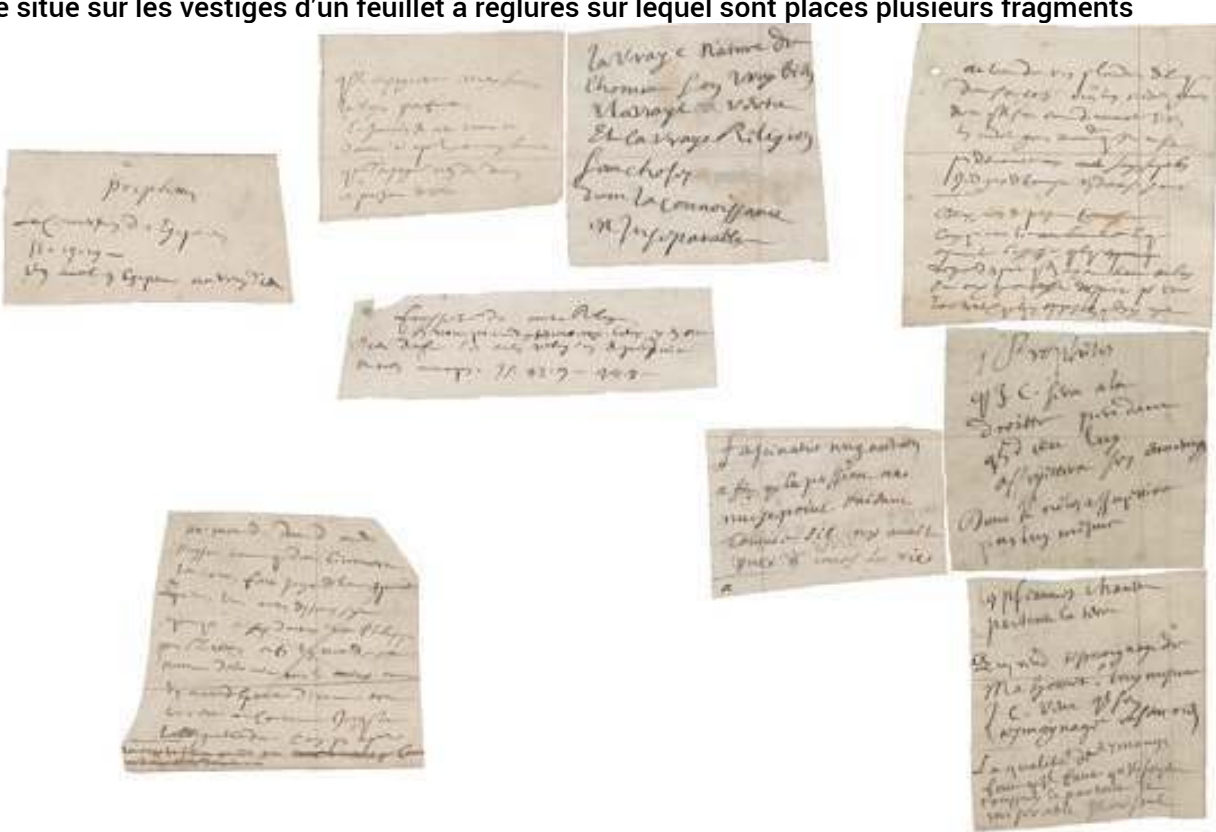

Les fragments : Prophéties L 330, Perpétuité L 279, Prophéties L 325, Fausseté L204, Série I, L 393, Série I, L 386, Série I, L 394, XXIV, L 624, Ordre, L1

Cliché Pol Ernst

Nota bene : rappelons que, pour citer le livre de P. Ernst, les références de l'édition Lafuma telles que l'auteur lui-même les donne sont conservées afin d'éviter les équivoques dues aux différences dans le découpage des fragments.

Le titre Ordre figure sur la copie et non sur l'original du fragment. Un papier volant du manuscrit a dû être perdu.

\section{Étude du sens du fragment}

- suematsu Hisashi, «Les Pensées et le métatexte », Équinoxe, 1, automne 1987, p. 27-53. Voir p. 45.

- Sur Sel. 37, et l'ordre de l'apologie en général, voir V. CARRAUD, Pascal et la philosophie, p. 93-102.

\section{Les psaumes chantés par toute la terre...}

Par a le sens local.

- SELLIER Philippe, Pascal et saint Augustin, p. 492. Sur les « psaumes chantés par toute la terre ». La mention des Psaumes s'explique par saint Augustin, pour qui ils sont le livre prophétique par excellence. Voir Confessions, IX, IV, 8, Bibliothèque augustinienne, t. 14, p. 84-85: « et tamen a toto orbe terrarum cantantur, et non est qui se abscondat a calore tuo »; trad. Arnauld d'Andilly, éd. P. Sellier, Folio, p. 301 : «mais ne se chantent-ils pas par toute la terre; et y a-t-il un lieu dans l'univers qui ne se sente de votre chaleur?». La formule signifie que la terre entière entend les prophéties messianiques et se trouve ainsi préparée à entendre la prédication catholique. 
- ERNST Pol, Approches pascaliennes, p. 36. Les Pères ont vu dans les Psaumes la description prophétique de la vie de Jésus-Christ, l'annonce de son incarnation, de la Passion et de la Résurrection: les Psaumes chantés par toute la terre sont donc un témoignage de JésusChrist.

\section{Qui rend témoignage de Mahomet ? Lui-même}

l'honneur de Kinji

1. on ne voit pas « $\mathrm{s}$ » sur l'original. Même les copistes ne l'ont pas vu.

2. La correspondance avec le fr. Sel. 342 n'était pas la lecture «misérables »: la qualité des témoins dont il s'agit dans Sel. 37 est d'un caractère général, alors que Sel. 342 porte sur la qualité spécifique des Juifs en tant que témoins de Jésus-Christ.

3. Le «et" avant "partout et misérables" serait nécessaire seulement pour insister sur « misérables». Or, «il [Mahomet] est seul » s'oppose à «toujours et partout », et non pas à « misérables ».

4. La construction rythmique du fragment favorise la lecture «misérable ": équilibre rythmique pour les lignes sur le christianisme ou sur la généralité et déséquilibre pour celles sur Mahomet.

Les psaumes / chantés // par toute / la terre. / (2 // 2)

Qui / rend témoignage / de Mahomet? // lui-même./ (3 // 1)

Jésus-Christ / veut // que son témoignage / ne soit rien./ (2 // 2)

La qualité / de témoins / fait // qu'il faut / qu'ils soient toujours / et partout,/ (3

// 3)

et, / misérable, // il est seul./ (2 // 1)

Tourneur, dans son édition paléographique, hésite et rétablit le s de misérable. Voir p. 245, n. 5 : « ce texte a été écrit par Pascal au crayon, sur une page de registre à filets rouges, 
puis repassé à l'encre par une main étrangère qui s'essaye tant bien que mal à suivre les traces de crayon en négligeant les barres de séparation qui indiquaient des intervalles entre les alinéas. (...) Il est probable qu'il faut restituer le signe s à la fin de misérable, bien qu'il ne soit pas tracé nettement par le crayon.»

L'édition Lafuma Luxembourg donne : «La qualité de témoins fait qu'il faut qu'ils soient toujours, et partout, et misérables. Il est seul. » Le mot misérables renvoie alors aux Juifs, témoins dispersés et misérables. Voir le commentaire critique de ERNST Pol, Approches pascaliennes, p. 35 et p. 684, met misérable au singulier, « conformément au manuscrit, et contrairement à la leçon de Tourneur (au pluriel), adoptée - on ne sait pour quelle raison -par Lafuma ».

L'édition de Philippe Sellier donne le même texte que Lafuma. Cette lecture est appuyée par la correspondance avec le fragment Sel. 342 - Laf. 311, où il est indiqué que c'est le peuple juif qui est misérable, "étant nécessaire pour la preuve de Jésus-Christ et qu'il subsiste pour le prouver et qu'il soit misérable, puisqu'ils l'ont crucifié. Et quoiqu'il soit contraire d'être misérable et de subsister, il subsiste néanmoins toujours malgré sa misère. "

L'interprétation de misérable au singulier n'est pas contradictoire avec le fait que le mot se rapporte au Juifs, si l'on admet la possibilité d'un accord selon le sens (avec le collectif peuple juif).

\section{Il est seul}

Y. Maeda note que le Il est seul est suivi d'un point d'exclamation dans l'édition Havet.

Voir la règle juridique énoncée dans DOMAT Jean, Les lois civiles, III, section III, § XIII. Un seul témoin ne fait pas preuve; il en faut au moins deux. C'est aussi un point de la loi juive.

\section{Sel. 38}

Ordre par dialogues.

"Que dois-je faire. Je ne vois partout qu'obscurités. Croirai-je que je ne suis rien?

Croirai-je que je suis dieu?»

« Toutes choses changent et se succèdent. »

«Vous vous trompez, il y a...»

«Et quoi ne dites-vous pas vous-même que le ciel et les oiseaux prouvent Dieu?» Non. «Et votre religion ne le dit-elle pas?» Non. Car encore que cela est vrai en un sens pour quelques âmes à qui Dieu donna cette lumière, néanmoins cela est faux à l'égard de la plupart.

Lettre pour porter à rechercher Dieu.

Et puis le faire chercher chez les philosophes, pyrrhoniens et dogmatistes, qui travailleront celui qui le recherche.

ERNST Pol, Géologie et stratigraphie..., p. 290. Papier : $\varepsilon$ feuillet à réglure. Voir la planche V, p. 424-425. Ordre, Laf. 2, 3 et 4 (Sel. 38). Moitié supérieure d'un feuillet 25 x 38 avec le filigrane à la marque royale. La moitié inférieure du feuillet n'a pas été retrouvée.

L'édition Sellier place un trait après le paragraphe qui s'achève par il y $a$. Dans le texte édité, les guillemets ont été ajoutés par les éditeurs, ce qui implique une interprétation. Le régime de la ponctuation des guillemets n'est pas toujours cohérent. Voir l'édition 
Sellier, où les non sont dépourvus de guillemets. L'édition Le Guern ne comporte pas de guillemets.

L'aspect du manuscrit suggère que Pascal a laissé des espaces blancs après certaines phrases, qui tenaient en quelque sorte lieu de titres de rubriques, comme «Vous vous trompez, il y a... », en attente de trouver les compléments nécessaires.

\section{Sel.38}

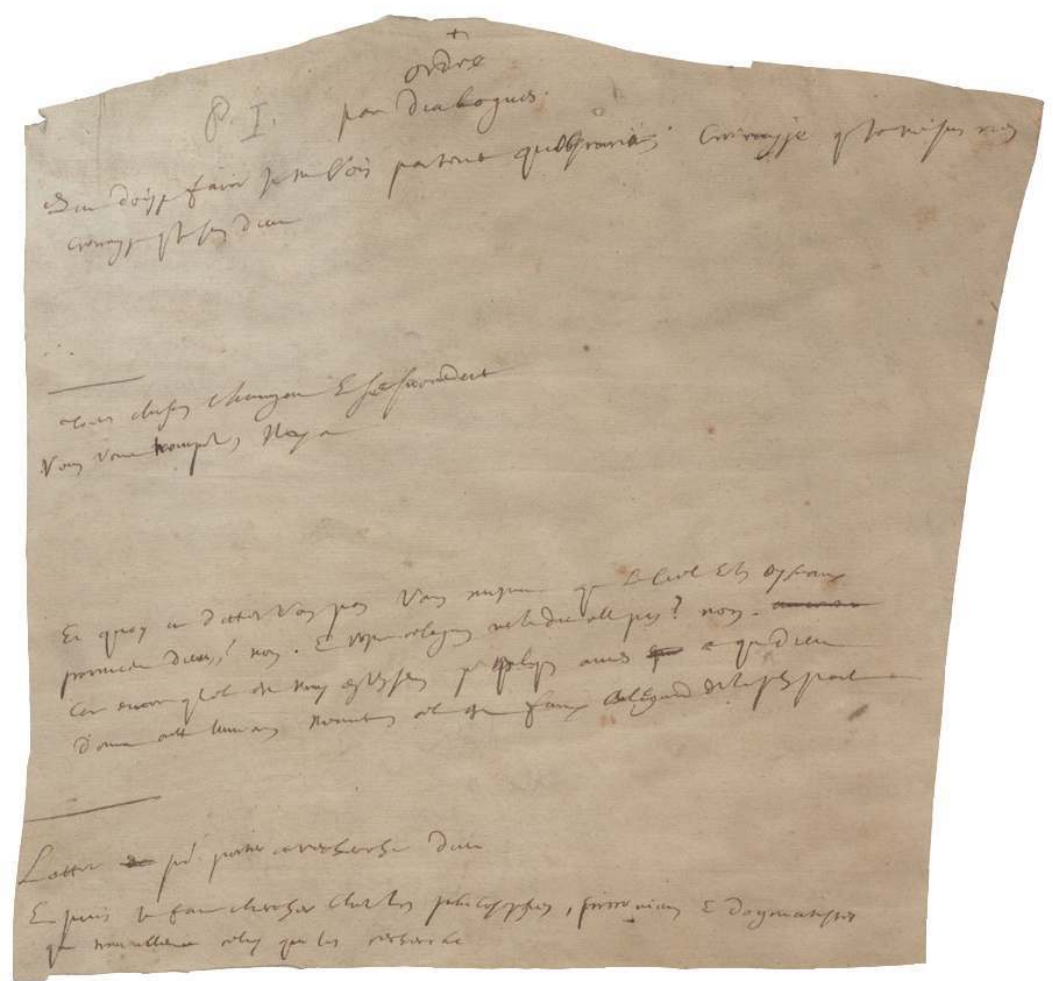

ERNST Pol, Géologie et stratigraphie..., p. 290. Papier : $\varepsilon$ feuillet à réglure. Voir la planche V, p. 424-425. Ordre, Laf. 2, 3 et 4 (Sel. 38). Moitié supérieure d'un feuillet $25 \times 38$ avec le filigrane à la marque royale. La moitié inférieure du feuillet n'a pas été retrouvée.

Cliché Pol Ernst

\section{Ordre par dialogues}

- BUSSON Henri, La pensée religieuse..., p. 587 sq. Le dialogue dans l'apologétique.

- harrington Thomas, "L'interlocuteur dans les Pensées de Pascal », Pascal, Port-Royal, Orient, Occident. Actes du colloque de l'Université de Tokyo, 27-29 septembre 1988, Klincksieck, Paris, 1991, p. 303-310.

- PARISH Richard, « Mais qui parle ? Voice and persona in the Pensées », p. 23-40.

- SELLIER Philippe, «Les premières Provinciales et le dialogue d'idées au XVII Siècle », Équinoxe, 6, été 1990, p. 21-30.

- SELLIER Philippe, Port-Royal et la littérature, I, Pascal, p. 74 sq.

- MARIN Louis, Pascal et Port-Royal, p. 24 sq.

- suematsu Hisashi, «Les Pensées et le métatexte », Équinoxe, 1, automne 1987, p. 27-53.

- suematsu Hisashi, "Voix dans le discours apologétique des Pensées ", Pascal, Port-Royal, Orient, Occident. Actes du colloque de l'Université de Tokyo, 27-29 septembre 1988, Klincksieck, Paris, 1991, p. 293-302. 
- VINET A., Études sur Blaise Pascal, p. 17 sq. «Il n'y a point de moi chez Pascal ; le héros, dirai-je, ou le patient de son livre, c'est l'homme ; et quand Pascal parle à la première personne, c'est qu'il se substitue, par procuration, au genre humain tout entier. Cette hardie personnification donne à son livre un caractère dramatique, bien rare dans un ouvrage de cette nature; ce livre, didactique en apparence, est tour à tour, suivant que le sujet le comporte, un drame, une véhémente satire, une philippique, une élégie, un hymne » : p. 19.

\section{Je ne vois partout qu'obscurités}

perbole : «je ne vois partout qu'obscurité » (au contraire selon Pascal dans le monde il n'y a pas une obscurité totale, mais un clair-obscur : les marques d'un Dieu caché, Sel. 229) ; « croirai-je que je ne suis rien? Croirai-je que je suis dieu? (La réponse de Pascal sera : "Connaissons donc notre portée. Nous sommes quelque chose et ne sommes pas tout ", Sel. 230) ; « Toutes choses changent et se succèdent » (selon Pascal plusieurs choses changent, mais pas toutes, si on veut considérer Dieu comme une « chose »).

\section{Croirai-je que je ne suis rien ? Croirai-je que je suis dieu?}

- Voir Sel. 230, Disproportion de l'homme.

- La Genèse, tr. Sacy, p. 157 sq. Sur le sens spirituel de ce passage.

- Voir Jansenius, Pentateuchus, Caput tertium Geneseos, p. 23. "Eritis sicut dii, non naturâ vel omnipotentiâ vel omniscientiâ, sed per dignoscentiam boni et mali »..., savoir « dignosceris non ex praeceptis alienis, tancent qui instructione egeatis, sed per vos ipsos propriâ industriâ, quid bonum, quid malum, quid appetendum quid fugiendum sit dignoscentes ". Augustin explique que Ève n'aurait pu le croire, s'il n'y avait déjà eu dans son esprit «amor quidam propriae potestatis", et "quaedam de se superba praesumptio, quae per illam tentationem fuerat convincenda et humilianda ». Le premier péché de l'homme a été la superbia, « seu amorem istius excellentiae».

- Voir Écrits sur la Grâce, Traité de la prédestination, 3, § 6, OC III, p. 793 : «Adam tenté par le Diable succomba à la tentation, se révolta contre Dieu, enfreignit ses préceptes, voulut être indépendant de Dieu et égal à lui. »

- Voir Sel. 751. «Eritis sicut dii scientes bonum et malum; tout le monde fait le Dieu en jugeant : cela est bon ou mauvais et s'affligeant ou se réjouissant trop des événements. »

- Le rapprochement est également indiqué avec Descartes, qui note que l'homme est semblable à Dieu par sa capacité de choix volontaire (Méditation IV).

- GOUHIER Henri, Blaise Pascal. Conversion et apologétique, p. 138. 


\section{Toutes choses changent et se succèdent.} a sans doute des idées dont il remet à plus tard la mise au point. La discussion porte sur ce qu'il était possible à Pascal d'ajouter, eu égard au relativisme radical qui s'affirme à ce point du dialogue.

51 « Vous vous trompez, il y a... ». Selon P. Serini, in B. Pascal, Pensieri, Pascal aurait continué cette pensée en opposant à l'incessant changement des choses l'immuable être de Dieu. Voir Sel. 167: « Mais je vois bien qu'il y a dans la nature un être nécessaire, éternel et infini ». Le rapprochement avec Sel. 38 et Sel. 167 conduit à un raisonnement de cette sorte : « Toutes choses changent et se succèdent » - « Vous vous trompez, il y a [...] dans la nature un être nécessaire, éternel, infini ». Dieu est le seul à ne pas changer (et, comme Lui, l'essentiel de la Révélation, voir liasse « Perpétuité »).

Voir l'édition de Martineau, qui associe par renvoi à Sel. 710, ce qui substitue au dialogue entre Pascal et l'incrédule un dialogue entre philosophes.

\section{Le ciel et les oiseaux prouvent Dieu}

- SELLIER Philippe, Port-Royal et la littérature, I, Pascal, p. 74 sq., renvoie à fr. S 644, et donne une référence au Ps. 18 (Coeli enarrant gloriam Dei).

- DROZ Edouard, Étude sur le scepticisme de Pascal, p. 91 sq. Droz interprète ce passage comme une allusion aux preuves physiques de Dieu.

- Le Guern renvoie à l'apologétique traditionnelle des humanistes, par exemple La théologie naturelle du P. Yves de Paris (mais pourquoi lui ?).

- Note de Paolo Serini : "Pascal avait pu lire dans le traité apologétique De l'immortalité de l'âme (1634) de Jean Silhon (1596-1667) un passage où on exaltait, suivant Montaigne (Essais, II, XII), la beauté des nids des oiseaux » (ibidem).

Courrier du Centre international Blaise Pascal, 24 | 2015 


\section{Coeli enarrant gloriam Dei (Psaume XVIII). Cela est faux à l'égard de la plupart}

Ce fragment est souvent rapproché de Sel. 644, 690.

- cousin Victor, Euvres, IV érie, Littérature, Blaise Pascal, p. 25. Texte invoqué à l'appui de la thèse selon laquelle Pascal «rejette toutes les preuves naturelles de l'existence de Dieu »: p. 24-25.

- PASCAL, Euvres complètes, éd. J. Mesnard, II, p. 582, lettre de Blaise et Jacqueline à Gilberte du $1^{\mathrm{er}}$ avril 1648. "Comme toutes choses parlent de Dieu à ceux qui le connaissent, ces mêmes choses le cachent à tous ceux qui ne le connaissent pas ».

- PASCAL, Euvres complètes, II, éd. Le Guern, p. 1314, renvoie là-dessus à ARNAULD Antoine, Apologie pour les saints Pères, IV, V, p. 205. "Ainsi ce sont deux choses qui s'accordent fort bien ensemble, que d'une part la beauté des créatures visibles soit un miroir éclatant, qui représente à tous les hommes l'invisible majesté de leur auteur, et les invite à l'adorer et à le servir; et que néanmoins le péché ayant rempli tous les hommes de ténèbres et d'aveuglement, il n'y ait plus personne depuis la corruption de la nature, qui étant laissé à lui-même, comme l'ont été les païens selon la parole sainte, se puisse servir de cette considération des créatures pour se porter à adorer Dieu, et à l'aimer plus que toutes choses, comme il est nécessaire pour le salut ».

\section{Quelques âmes à qui Dieu donna cette lumière}

- Lecture de Havet : «Dieu donne » au lieu de «Dieu donna ».

- SELLIER Philippe, Port-Royal et la littérature, I, Pascal, p. 75. Formule interprétée comme une allusion aux platoniciens, en supposant que cette lumière est naturelle.

\section{Lettre pour porter à rechercher Dieu. Et puis le faire chercher chez les philosophes, pyrrhoniens et dogmatistes qui travailleront celui qui le recherche.}

- MAEDA Yoichi, Commentaire des Pensées, I, p. 9 sq.

- tourneur, Pensées, éd. paléographique, p. 167.

- SELLIER Philippe, Port-Royal et la littérature, I, Pascal, p. 55 sq. La lettre pour porter à chercher Dieu se situe avant l'enquête anthropologique : p. 56.

- Pensées, éd. Havet, II, p. 144, n 108 bis. Mis en addition du fragment Sel. 573. Édition Havet : pas de nouvelle ligne pour « Une lettre »; «chercher, et il répondra » au lieu de « chercher. Et il "; un virgule après « mais que » et après « de rien ».

- DROz Édouard, Étude sur le scepticisme de Pascal, passim.

- vinet A., Études sur Blaise Pascal, p. 74 sq. Du livre de M. Cousin sur les Pensées de Pascal. Pascal « ne professe pas le pyrrhonisme, mais... il le constate ; ... il signale la tempête des opinions, le trouble des intelligences, la détresse de l'esprit humain, pour faire sentir combien il était urgent que Dieu fît tomber dans ces vastes et déplorables ténèbres ce rayon de sa gloire... et que nos cœurs émus appellent Jésus-Christ ", p. 100. Face aux pyrrhoniens, Pascal ressent un mélange de mépris et de terreur ; désordre méprisable, mais qui emporte tout : p. 187-188. Ils tirent leur force d'une pétition de principe: p. 187. Pascal considère le pyrrhonisme comme une maladie de l'esprit, mais inhérente à l'homme, suite de notre déchéance : p. 193. 


\footnotetext{
- MEURILlon Christian, «La lettre au cœur de l'écriture pascalienne», Revue des Sciences humaines, 195, 1984-3, p. 5-18.

- DUCHENE Roger, L'imposture littéraire dans les Provinciales.

- SELLIER Philippe, «Vers l'invention d'une rhétorique », in SELLIER Philippe, Port-Royal et la littérature, I, Pascal, Champion, Paris, 1999, p. 171 sq.

- ADAM Antoine, Histoire de la littérature française au XVII siècle, I, p. 243.

- fumaroli Marc, L'Age de l'éloquence, p. 154 sq.

- ferreyrolles Gérard, Les Provinciales, p. 14 sq.

- fumaroli Marc, «Genèse de l'épistolographie classique : rhétorique humaniste de la lettre, de Pétrarque à Juste Lipse », Revue d'Histoire Littéraire de la France, nov.-déc. 1978, p. 886-900.
}

\section{... Qui travailleront celui qui le recherche}

Lecture de Tourneur, édition paléographique, p. 167 : celui qui les recherche. En note: «Peut-être pour le sens vaudrait-il mieux lire le, malgré le ms; car il arrive parfois que Pascal écrive ainsi l'e final. » Tourneur : travaillent ou travailleront.

Lecture de Havet: «qui travaillent » au lieu de «qui travailleront». Dans Havet 1852 : celui qui le recherche. À partir de la 4 e édition, pluriel les. Havet a voulu intégrer Molinier.

\section{Sel. 39}

Ordre. 
Une lettre d'exhortation à un ami pour le porter à chercher. Et il répondra : « Mais à quoi me servira de chercher, rien ne paraît. " Et lui répondre : «Ne désespérez pas. » Et il répondrait qu'il serait heureux de trouver quelque lumière. Mais que selon cette religion même quand il croirait ainsi cela ne lui servirait de rien. Et qu'ainsi il aime autant ne point chercher. Et à cela lui répondre : « La machine ».

\section{Établissement du texte}

- ERnST Pol, Les Pensées de Pascal. Géologie et stratigraphie, p. 231. Strate FNIC.

- maeda Yoichi, Commentaire des Pensées, I, p. 9 sq. Il y a eu deux étapes dans la rédaction; la première ligne a été ajoutée après le reste, et même après le titre. Pascal écrit d'abord: «Une lettre où un ami dise : Mais à quoi me servira de chercher... » Il barre " une lettre où un ami dise » et écrit au-dessus: "une lettre d'exhortation à un ami pour le porter à chercher. Et il répondra... » Le dernier mot, La machine, est aussi postérieur.

Y. Maeda remplace le mot croirait ainsi par crierait.

\section{Fragments connexes}

- Sel. 142.

- Sel. 225.

- Sel. 240 .

- Sel. 277 : «Objection des athées : « Mais nous n'avons nulle lumière » ».

- Sel. 384 .

- Sel. 655 .

- Sel. 681.

\section{Exhortation}

61 Sur la nécessité d'un interprète bienveillant pour s'initier à la religion, voir :

- saint Augustin, De utilitate credendi, VI, 13, p. 237 sq.

- KIBEDI-VARGA Aron, Rhétorique et littérature, p. 29. L'exhortation rangée sous le genre délibératif, avec les remontrances, les conseils et les consolations.

\section{L'objection de l'incroyant}

- MESNARD Jean, Les Pensées de Pascal, p. 241. Sur Fondement de la religion et réponse aux objections . L'objection est nous n'avons nulle lumière.

- SELLIER Philippe, Port-Royal et la littérature, I, Pascal, p. 61. L'idée repose sur le principe que « la foi est différente de la preuve », Sel. 41. Voir sur ce point l'édition Sellier qui renvoie à Saint Thomas, Somme de Théologie, IIa II ae q. 1, a 5 pour le commentaire.

Le rapprochement effectué par M. Le Guern (Paris, Gallimard, 1977) avec le fragment Sel. 277 de la liasse Fondements de la religion et réponse aux objections : "Objection des athées : Mais nous n'avons nulle lumière ", semble indiquer que l'ami est un athée.

- SHIOKAWA Tetsuya, "Justus ex fide vivit et fides ex auditu: deux aspects de la foi dans l'apologétique pascalienne », in MEURILLon Christian (dir.), Pascal, l'exercice de l'esprit, Revue des sciences Humaines, 244, 1996, p. 159-178.

La discussion porte sur le fait que si le fragment Sel. 39 répond à Sel. 38, par l'idée de commencer par une «Lettre pour porter à chercher Dieu », après cette «Lettre ", il 
semble y avoir une discordance. Sel. 38 propose de «le faire chercher chez les philosophes ". C'est le parcours des liasses de la première colonne, suivant la logique de l' Entretien. Après ce parcours, «A.P.R. » on présente le point de vue chrétien (déjà anticipé dans Contrariétés, Sel. 164). Sel. 39 propose l'objection de l'ami à propos de l'utilité des preuves. Ce parcours est soutenu dans les fragments Sel. 41, Sel. 45, mais il n'a pas été suivi dans les liasses à titre. Pour accorder cette contradiction, l'idée est proposée que la recherche chez les philosophes fait partie de la lettre pour porter à chercher de laquelle parle Sel. 39. Il faudrait donc envisager le parcours suivant :

1. Une «lettre pour porter à chercher». Dans cette lettre il y a la recherche chez les philosophes.

2. Après la recherche chez les philosophes, l'objection de l'ami: les philosophes n'ont découvert aucune vérité ; donc, " à quoi me servira de chercher ? Rien ne paraît ».

Il y a une deuxième possibilité, suivant l'hypothèse de P. Sellier : Ordre rassemble des notes où Pascal, lors de la constitution des vingt-six liasses provisoires de l'Apologie, a indiqué les projets nouveaux qui germaient en lui (Paris, Garnier, 1999, p. 164, n.1). En ce cas, la discordance entre Sel. 38 et Sel. 39 ne pose aucun problème: Pascal envisageait différentes possibilités et les notait dans Ordre.

\section{Rien ne paraît ; le manque de clarté}

M. Le Guern (Paris, Gallimard, 1977) renvoie au fragment Sel. 277 de la liasse Fondements de la religion et réponse aux objections: «Objection des athées : «Mais nous n'avons nulle lumière ». L'ami est donc un athée. Cette pensée se trouve dans la liasse Fondements: le principe pour répondre aux objections y est que Dieu « a voulu aveugler les uns et éclairer les autres » (Sel. 264), donc si on le conteste on ne démontre pas que Dieu n'existe pas, mais qu'on est aveuglé.

Autres renvois : Sel. 260 : «Qu'on ne nous reproche donc plus le manque de clarté, puisque nous en faisons profession »; Sel. 266 : «Dieu veut plus disposer la volonté que l'esprit. La clarté parfaite servirait à l'esprit et nuirait à la volonté. Abaisser la superbe » (L'abaissement de la superbe est un élément essentiel du "Discours de la machine», indiqué à la fin de Sel. 39) ; Sel. 267 : « Jésus-Christ est venu aveugler ceux qui voient clair et donner la vue aux aveugles... »; Sel. 268 : « Il y a assez de clarté pour éclairer les élus et assez d'obscurité pour les humilier. Il y a assez d'obscurité pour aveugler les réprouvés et assez de clarté pour les condamner et les rendre inexcusables »; Sel. 274 : «Dieu [...] a donné des marques de soi visibles à ceux qui le cherchent et non à ceux qui ne le cherchent pas ».

\section{II serait heureux de trouver quelque lumière}

67 Voir les deux versions de la Vie de Pascal, OC I, § 53, p. 622 : «il voulait auparavant connaître s'ils cherchaient la vérité de tout leur cœur ».

Noter que la nécessité du désir de vérité dans la recherche est un thème platonicien. 


\section{Ne désespérez pas}

69 Sur le désespoir en général, voir Sel. 225 : «La connaissance de sa misère sans celle de Dieu fait le désespoir »; Sel. 240: «S'ils reconnaissent l'infirmité de la nature, ils en ignorent la dignité, de sorte qu'ils pouvaient bien éviter la vanité, mais c'était en se précipitant dans le désespoir »; Sel. 384 : « La misère persuade le désespoir ».

Sur le désespoir des athées, voir Sel. 690 : «le désespoir des athées, qui connaissent leur misère, sans Rédempteur ».

Ce sont aussi les pyrrhoniens à souligner la misère de l'homme (voir par exemple l'Entretien avec M. de Sacy: le pyrrhonien Montaigne établit la «faiblesse » de l'homme, p. 125 de l'éd. Mengotti-Mesnard, Paris, Desclée de Brouwer, 1994).

Remarques proposées au cours du séminaire : le fragment Sel. 690 : (« le désespoir des athées, qui connaissent leur misère, sans Rédempteur »), qui se réfère explicitement à Sel. 277 (on parle des athées) et à Sel. 225, Sel. 240 et Sel. 384, fait une sorte de synthèse entre deux désespoirs :

1. le désespoir de ceux qui disent qu'on ne peut rien trouver (Sel. 277 : « Mais nous n'avons aucune lumière »; Sel. 39 : « Rien ne paraît »)

2. le désespoir de ceux qui, après avoir cherché, ont trouvé que l'homme est misérable, sans avoir trouvé Dieu (Sel. 225, Sel. 240, Sel. 384).

L'objection des athées " rien ne paraît ", correspond à " nous n'avons nulle lumière ", de sorte que même si l'on cherche, on ne trouve rien, la réponse de Pascal « Ne désespérez pas » renvoie au fondement de la religion : « Dieu a tempéré sa connaissance en sorte qu'il a donné des marques de soi visibles à ceux qui le cherchent et non à ceux qui ne le cherchent pas" (Sel.274; voir la dernière partie de Sel.182). Voir aussi Sel.190 (Commencement), où Pascal reproche à l'ami de négliger les signes. « Ne désespérez pas » signifie donc « Dieu a donné des marques de soi visibles à ceux qui le cherchent ».

\section{À quoi me servirait de chercher ? Il aime autant ne point chercher}

SELLIER Philippe, Port-Royal et la littérature, I, Pascal, p. 61 sq. La lettre sur l'utilité des preuves.

Ce fragment rapproche donc implicitement pyrrhoniens et athées : les uns et les autres connaissent la misère de l'homme et ignorent le Rédempteur. L'identité pyrrhonienne de l'ami est indiquée aussi par la suite du fragment : Pascal ne dit pas que l'ami « préfêre ne point chercher», mais qu'il «aime autant ne point chercher» (le pyrrhonien tend à l'indifférence, donc il ne « préfère » pas : il « aime autant »).

\section{Quand il croirait ainsi, cela ne lui servirait de rien.}

Havet : en note pour « ainsi » : C'est-à-dire sans se convertir de cœur, sans se sanctifier.

Remarque proposée au cours du séminaire: "Croirait ainsi » signifie "croire à cause des preuves, par raisonnement, sans sentiment, sans foi ». Pascal explique en quoi c'est vrai dans Sel. 142: «Ceux à qui Dieu a donné la religion par sentiment de cœur sont bien heureux et bien légitimement persuadés. Mais à ceux qui ne l'ont pas nous ne pouvons la donner que par raisonnement, en attendant que Dieu la leur donne par sentiment de 
cœur. Sans quoi la foi n'est qu'humaine et inutile pour le salut», et Sel. 412: "On ne croira jamais, d'une créance utile et de foi, si Dieu n'incline le cœur. Et on croira dès qu'il l'inclinera ». Donc, l'ami a raison : la religion par raisonnement, que Pascal donnerait par le moyen des preuves, est inutile pour le salut, sans le sentiment de cœur que Dieu seul peut donner.

Mais l'ami a aussi tort, parce qu'il n'a considéré que deux moyens de croire : l'inspirationsentiment (qui selon lui est la seule qui compte) et la raison (qui selon lui est inutile pour le salut). Le fragment Sel. 655 répond à l'objection de l'ami en indiquant un troisième moyen de croire : « la machine » : « Il y a trois moyens de croire : la raison, la coutume, (l') inspiration. La religion chrétienne, qui seule a la raison, n'admet point pour ses vrais enfants ceux qui croient sans inspiration. Ce n'est pas qu'elle exclue la raison et la coutume, au contraire; mais il faut ouvrir son esprit aux preuves, s'y confirmer par la coutume, mais s'offrir par les humiliations aux inspirations, qui seules peuvent faire le vrai et salutaire effet, ne evacuetur crux Christi » (I, Corinthiens, 1, 17).

Donc, à l'objection : « Croire sans inspiration ne sert de rien » Pascal répond :

1. Vous avez raison : selon cette religion même, seules les inspirations « peuvent faire le vrai et salutaire effet »;

2. Mais vous avez tort: selon cette religion même, "il faut ouvrir son esprit aux preuves " (voir « lettre qui marque l'utilité des preuves », résumée dans Sel. 41) et « s'y confirmer par la coutume » (voir « discours de la machine », Sel. 680).

\section{La machine}

Dans l'édition Le Guern, l'initiale de « Machine » est majuscule dans la liasse « Ordre » et minuscule dans « Vanité » (Sel. 59) et « Raisons des effets » (Sel. 118).

La discussion de fond sur la machine est remise à un examen ultérieur.

\section{Sel. 40}

Première partie. Misère de l'homme sans Dieu. Deuxième partie. Félicité de l'homme avec Dieu. Autrement Première partie. Que la nature est corrompue, par la nature même. Deuxième partie. Qu'il y a un Réparateur, par l'Écriture. 


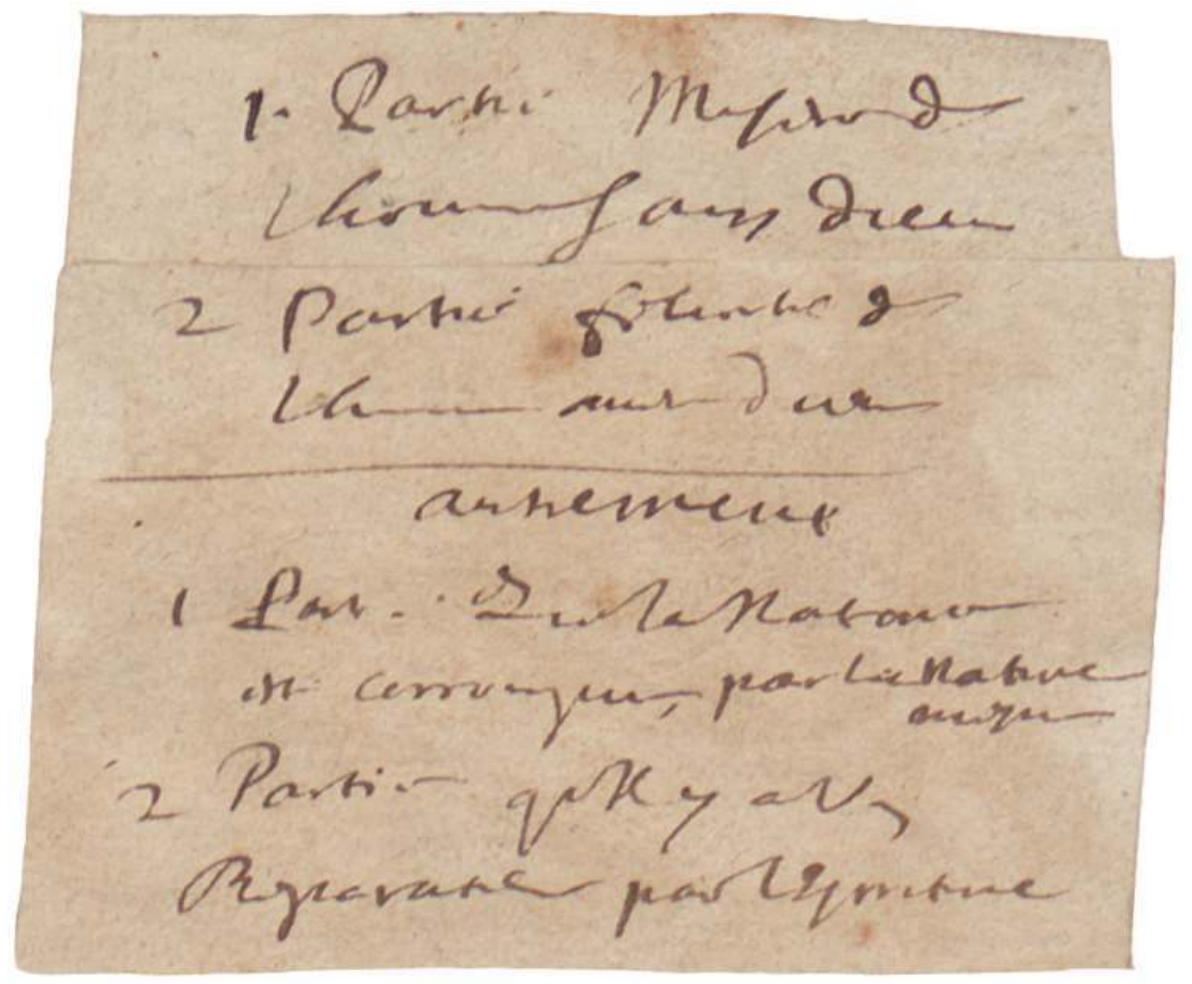

Ernst Pol, Géologie et stratigraphie..., p. 290. Papier non identifié.

Crédit Ernst Pol

Havet : «seconde partie » au lieu de "deuxième partie »; pas de séparation, ni de nouvelle ligne pour «Autrement» «.Par l'Écriture» au lieu de «,par l'Écriture». Dans la $1^{\text {ère }}$ éd., en note pour « Première partie » : Non reproduit dans les éditions. C'est une division du plan que Pascal méditait. Cf. VI, 33 [" Parler de ceux qui ont traité »: Sel. 644].

La reconstitution de Y. Maeda, t. 1, p. 32, montre que la disposition sur le manuscrit est la suivante (les additions sont en gras) :
1. Partie. Misère de
l'homme sans Dieu.
2. Partie. félicité de
l'homme avec Dieu.
Autrement
1. Part. Que la nature
est corrompue, par la Nature
même.
2. Partie. qu'il y a un
Réparateur par l'Écriture.

84 C'est une sorte de calligramme. Leur situation dans le manuscrit met en correspondance les éléments corrélatifs, comme dans un tableau à double entrée, comme il y en a souvent chez Pascal (Generatio conisectionum, Traité de la prédestination).

Pascal prévoit des parties (le terme de partie est rarement employé dans les textes achevés de Pascal) chacune répondant à une voie vers la vérité. "Par» a une signification 
méthodologique et renvoie aux sources de la connaissance humaine; voir Gouhier, Commentaires, p. 168-169.

- FORCE Pierre, Pascal, Pensées, p. 20 sq. Point commun des deux plans : la première partie ne fait pas référence à la vérité révélée.

- LACOMBe Roger, L'apologétique de Pascal, p. 33-36. Hypothèses sur les hésitations de Pascal sur son ordre.

Le Guern renvoie au fragment LG 434. Pour lui, la deuxième partie du fragment, «cette seconde ébauche de division correspond en gros au plan adopté par Pascal pour le classement en liasses ».

Selon J.R. Cole (Pascal's Plans for the Apology, "French Studies ", n 1, 1996, pp. 15-34) Sel. 40 est en contradiction avec Sel. 46. Pascal d'abord aurait envisagé une Apologie en trois parties (1- «montrer que la religion n'est point contraire à la religion»; 2- «la rendre ensuite aimable »; 3- «montrer qu'elle est vraie»), selon le programme de Sel. 46 suivi dans la colonne de droite. Après cela Pascal aurait envisagé une Apologie en deux parties (1- « misère de l'homme sans Dieu »; 2- «félicité de l'homme avec Dieu »), selon le programme de S.40. La «Table » représente « Pascal's decision to shift from the threestage scheme of Pensée 12 [Sel. 46] to the two-part plan of Pensée 6 [Sel. 40] » (p. 32).

- Ferreyrolles Gérard, «De l'usage de Senault: apologie des passions et apologétique pascalienne ", Corpus, 7, 1988, p. 3-20. Rapproche l'esquisse de Pascal du plan des titres de deux livres de Senault, L'homme criminel, ou la corruption de la nature par le péché, 1644, et L'homme chrétien, ou la réparation de la nature par la grâce, 1648.

- PÉRIER Gilberte, Vie de Pascal, OC I, p. 621. « Il croyait qu'il ne fallait jamais affliger qu'on ne consolât, et que bien ménager tout cela était le secret de l'éloquence ».

- DOMPNIER Bernard, «Pastorale de la peur et pastorale de la séduction", in La conversion au XVII siècle, p. 264 sq. On ne convertit jamais le pécheur en le désespérant : p. 274.

- RUSSIER Jeanne, La foi selon Pascal, I, p. 34 sq. Ne pas prendre pour démonstration ce qui est tactique, «envisageant moins la vérité que l'opportunité », p. 40. «Peu importe la manière, ce qui compte, c'est le résultat, l'abaissement », p. 40. «Il est question non de démontrer, mais de convertir», p. 40. "Cure mentale» dont Pascal emprunte les instruments à Montaigne. Il importe moins de réfuter que de confondre, de prendre l'offensive pour retourner la situation : p. 43.

- GOUhier Henri, Blaise Pascal. Commentaires, p. 168 sq.

- Sellier Philippe, Port-Royal et la littérature, I, Pascal, p. 67 sq.

- suematsu Hisashi, «Les Pensées et le métatexte », Équinoxe, 1, automne 1987, p. 27-53.

\section{Échos et fragments connexes}

- Sel. 35. Opposition avec/sans Jésus-Christ.

- Sel. 681. Par eux-mêmes.

- Sel. 644, par le rapprochement avec Montaigne «sans Dieu », qui en fait une extension de Sel. 40.

88 Misère renvoie à un titre de liasse, de même que Nature corrompue.

\section{Félicité de l'homme avec Dieu}

MESNARD Jean, Les Pensées de Pascal, $2^{e}$ éd., p. 219. Pascal oppose en général «grandeur » à « misère ». Ici, il oppose "félicité » à « misère »: dans le premier couple, l'homme est 
envisagé en lui-même et sa double nature; dans le second, il est mis en rapport avec sa fin. Relation avec les deux parties que Pascal a prévues, l'une qui a l'homme pour centre, dans laquelle Dieu n'intervient pas, sinon comme solution possible aux contradictions; le seconde, marquée par la relation entre l'homme et Dieu. En considérant seulement la première colonne, l'opposition majeure est celle de la misère et de la grandeur. Dans ce couple, «le second terme [...] ne fait que désigner une autre face du premier ». Après Le Souverain Bien, Pascal change de point de vue : la coprésence de misère et grandeur dans l'homme montre que l'homme est misérable (c'est-à-dire "malheureux ») sans Dieu : les dix liasses de la première colonne sont organisées "autour du thème de la misère ", entendue au sens contraire de félicité. L'opposition "misère/félicité » se trouve aussi dans Sel. 35 (la « liasse table ») où, au lieu de « sans Dieu / avec Dieu » nous avons « sans J.-C./avec J.-C. ». «Sans J.-C. » l'homme est dans le vice et dans la misère. «En J.-C. » est toute notre vertu et toute notre félicité. « Vertu » est le contraire de "vice »; « misère » est le contraire de « félicité ».

Si on considère la totalité de l'Apologie, la colonne de gauche marque la misère de l'homme et la colonne de droite la félicité de l'homme : « Des dix chapitres de la première partie on peut donc dire qu'ils sont organisés autour du thème de la misère, entendue au sens contraire de félicité. [...] Dans la perspective de l'homme avec Dieu, c'est-à-dire au cours de la seconde partie, [...] la religion chrétienne sera présentée comme seule capable de répondre aux besoins et aux aspirations de l'homme " (J. Mesnard, op. cit., p. 220). On remarque pourtant que, dans la colonne de droite, une liasse semble n'avoir rien à voir avec la félicité de l'homme : La nature est corrompue. Mais selon l'hypothèse de J. Mesnard cette liasse, avant d'être supprimée par Pascal, ne comprenait que deux fragments: Sel. 35 et Sel. 36 (J. Mesnard, op. cit., pp. 44-6). Or, Sel. 35 marque l'importance morale pour l'homme de la venue de J.-C., Sel. 36 marque son importance au niveau de la connaissance. L'hypothèse de J. Mesnard est donc cohérente: si ces deux fragments constituent le contenu de La nature est corrompue, alors la colonne de droite peut être considérée la deuxième partie où Pascal montre la félicité de l'homme avec Dieu.

\section{Sel. 41}

Lettre qui marque l'utilité des preuves. Par la Machine.

La foi est différente de la preuve. L'une est humaine, l'autre est un don de Dieu. Justus ex fide vivit. C'est de cette foi que Dieu lui-même met dans le cœur dont la preuve est souvent l'instrument. Fides ex auditu. Mais cette foi est dans le cœur et fait dire non Scio mais Credo.

Sel.41

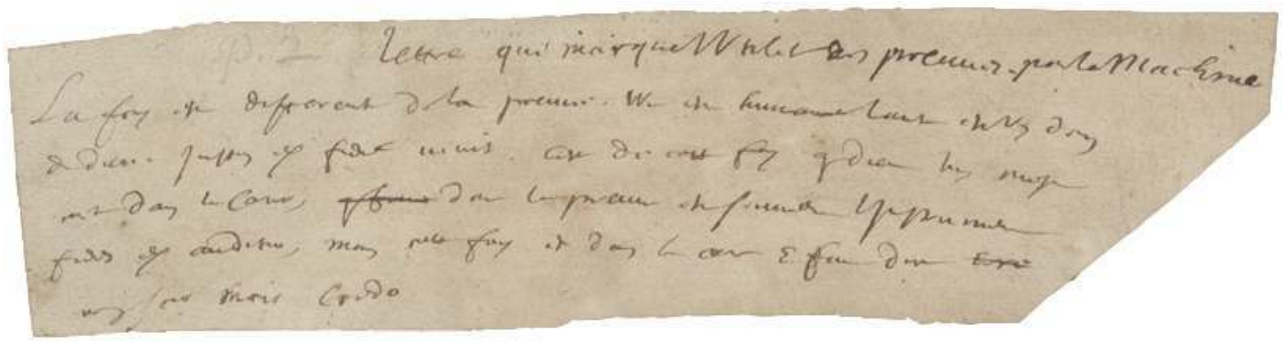




\section{Genèse du texte}

- MAEDA Yoichi, Commentaire des Pensées, I, p. 35.

- ERNST Pol, Les Pensées de Pascal. Géologie et stratigraphie, p. 231. Strate FNIC.

\section{Lettre qui marque l'utilité des preuves. Par la machine.}

Remarque de l'édition Havet: «Lettre qui marque l'utilité des preuves par la machine.» Est-ce un jésuite qui parle ainsi, ou Pascal? Port-Royal a bien fait de supprimer ces derniers fragments, et de ne pas trop laisser paraître l'effort où se pliait ce génie violent, mais violent contre lui-même. »

\section{La foi est différente de la preuve. L'une est humaine, l'autre est un don de Dieu}

\section{Fides ex auditu : Romains, X, 17.}

- Dans l'édition Havet, en note, renvoi à Rom. X, 17. Traduction de Sellier : " La foi vient de ce qu'on a entendu ». Le Guern: «La foi vient par l'audition ». Traduction de Sacy: «La foi donc vient ce qu'on a ouï ».

- shiokawa Tetsuya, "Justus ex fide vivit et fides ex auditu: deux aspects de la foi dans l'apologétique pascalienne », in MEURILLon Christian (dir.), Pascal, l'exercice de l'esprit, Revue des sciences Humaines, 244, 1996, p. 159-178.

- SELLIER Philippe, Port-Royal et la littérature, I, Pascal, p. 61 sq.

Remarque: Après la preuve, un autre instrument humain utile pour la foi est le témoignage (auditus). Pascal annonce un thème qu'il développera dans Conclusion: l' auditus est instrument de la foi car «Ceux qui croient sans avoir lu les Testaments, c'est parce que : 1 . ils ont une disposition intérieure toute sainte $; 2$. ce qu'ils entendent dire de notre religion y est conforme » (Sel. 413). Le fragment Sel. 413 est fondé sur la distinction « Ils sentent » (l'inspiration divine) / «Ils entendent dire » (le témoignage de l'Église). Pascal ne peut provoquer l'inspiration divine, ni la fidem, mais il peut exposer les aspects essentiels du christianisme, donner l'auditum. Après la lecture des Pensées, le lecteur $a$ entendu. Si Dieu lui donne la disposition sainte du cœur, il peut considérer la conformité entre ce qu'il sent et ce qu'il a entendu. 
Remarque : à propos du principe fides ex auditu et sur le problème du témoignage qu'il désigne. Il semble que le fragment Sel. 672 («L'autorité») prolonge et déplace la problématique du fragment 41 vers la question de la légitimité du témoignage: "Tant s'en faut que d'avoir ouï dire une chose soit la règle de votre créance, que vous ne devez rien croire sans vous mettre en l'état comme si jamais vous ne l'aviez oui. / C'est le consentement de vous à vous-même et la voix constante de votre raison, et non des autres, qui vous doit faire croire ». À Port-Royal, la formule fides ex auditu est d'ailleurs au centre du débat sur le pouvoir ecclésiastique et ses abus; elle sera notamment au centre du Traité de la foi humaine en 1664. Enfin et hors de ce contexte polémique, deux références bibliques (Bible de Sacy) semblent désigner ce même «au-delà » du témoignage. Voir Job 42, 5: "Mon oreille auparavant vous avait entendu, mais maintenant je vous vois de mes propres yeux»; et Psaume 48 (1-5 surtout). Avant d'ouvrir le cœur ou de le toucher, si celui-ci y est disposé, le témoignage doit subir un examen rationnel ; après, qu'il ait ouvert la voie à l'inspiration ou non, il doit disparaître ; l'auditum n'a que la consistance précaire de la voix qui le porte.

\section{Scire et credere}

- Renvoi à Sel. 409: "Qu'il y a loin de la connaissance de Dieu à l'aimer ». L'identification entre «Scio» (S.41) et "connaissance " (S.409) va de soi. L'identification entre «Credo» (S.41) et « aimer Dieu » (Sel. 409) est prouvée par Sel. 412 : « Dieu leur donne l'amour de soi [... ], il incline leur cœur à croire».

- Note de Alberto Moscato: "Pour la différence entre scire et credere, on peut rappeler le tacitien sanctius ac reverentius videri de actis deorum credere, quam scire, très souvent cité. Entre autres, y fait appel, à l'appui d'une apologétique sceptique, La Mothe Le Vayer, Deux dialogues ...par Orasius Tubero. I. Sur la divinité, éd. Tisserand, Paris, 1922, p. 147 » (in B. Pascal, Antologia filosofica, a cura di A. Moscato, La Scuola, Brescia, 1982).

- Note de M. Le Guern : «Cette distinction est un des éléments les plus constants de la pensée de Pascal. Elle est déjà formulée dans la Préface sur le traité du vide, composée aux alentours de 1650, qui oppose les connaissances fondées sur la raison et l'expérience à celles qui relèvent de l'autorité, au premier rang desquelles il convient de placer la théologie ».

L'édition Sellier renvoie à S. Thomas, Somme de Théologie, IIa II ae q. 1, a 5 pour le commentaire. «Utrum ea quae sunt fidei possint esse scita ».

100 La formule paulinienne scio cui credidi figure sur le sceau adopté par Pascal après sa conversion.

\section{L'autre est un don de Dieu}

MESNARD Jean, Les Pensées de Pascal, 2e éd., p. 325.

\section{La preuve est souvent l'instrument}

Selon le programme de Sel.579, maintenant il faut corriger l'ami. Celui-ci objecte que, puisque la preuve n'est pas toujours utile, elle ne sert de rien. Pascal répond qu'il ne voit pas que souvent la preuve est l'instrument de la foi, donc qu'il ne faut pas mépriser les preuves, car Dieu souvent s'en sert pour donner la foi. 
Le rapport entre « inspiration divine » et " preuve humaine » rappelle le rapport entre « cause première » et « cause seconde » des Écrits sur la grâce:

1. l'inspiration divine est la cause première (indispensable) de la conversion,

2. la preuve humaine est la cause seconde.

Un exemple d'utilité de cette « cause seconde » est le dialogue avec l'infidèle. Toutes les religions ont des croyants par sentiment. Mais « la religion chrétienne [...] seule a la raison » (Sel. 655), c'est-à-dire qu'elle est la seule à avoir les preuves. Cette supériorité ne peut pas être montrée par « un de ces chrétiens qui croient sans preuves » (Sel. 414), mais seulement par «ceux qui savent les preuves» (S.414). Donc, le chrétien « habile » peut montrer à l'infidèle « demi-habile » que le « simple peuple » chrétien est vraiment incliné par Dieu.

\section{Sel. 42}

Ordre.

Voir ce qu'il y a de clair dans tout l'état des Juifs et d'incontestable.

Sel.42

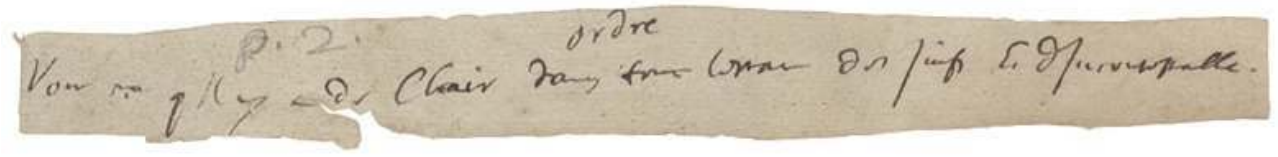

ERNST Pol, Les Pensées de Pascal. Géologie et stratigraphie, p. 232. Strate FNIC.

Crédit Pol Ernst

Dans ce fragment, le titre Ordre est une addition.

Suematsu Hisashi, «Les Pensées et le métatexte », Équinoxe, 1, automne 1987, p. 27-53. Voir p. 46, sur le fait que ce fragment annonce les dossiers sur L'État des Juifs.

\section{Fragments associés}

- Sel. 342.

- Sel. 646.

- Sel. 696.

- Sel. 734.

- Sel. 736 (« visiblement »).

Le mot voir peut être entendu en deux sens distincts, selon qu'on l'interprète comme l'expression d'un projet de l'auteur, ou comme l'expression elliptique d'un conseil adressé à un lecteur. Dans les deux cas, il faudrait suppléer il faut voir...

Ce qui est incontestable est indiqué dans les fragments mentionnés ci-dessus : que les Juifs sont toujours, et partout, etc.

Pour la démarche qui consiste à passer de l'incontesté au contesté, voir la Lettre sur la possibilité des commandements, 2, § 34, Écrits sur la grâce, OC III, p. 657 ; mais incontestable n'est pas équivalent d'incontesté. Incontestable s'entend d'une part au sens de incontestable par des hommes, ennemis des Juifs ; d'autre part incontestable par les textes, autrement dit 
dans le domaine de l'histoire. Une telle expression exclut que Pascal soit effectivement sceptique en histoire.

\section{État (des Juifs)}

Dictionnaire de l'Académie: disposition dans laquelle se trouve une personne, une chose, une affaire. Mauvais, heureux, malheureux, pitoyable état. Je laissai l'armée en bon état. Il a envoyé savoir l'état de votre santé (...). Il est dans un état à faire pitié. (...). On appelle État du ciel la disposition où se trouvent tous les astres les uns à l'égard des autres dans un certain moment (...). On dit Être en état de grâce. Être en état de péché mortel. L'état de perfection. L'état de grâce. L'état d'innocence. L'état de péché. (...) État signifie aussi la manière somptueuse, simple ou modeste dont on s'habille. Les bourgeoises portent aujourd'hui un aussi grand état que les femmes de qualité. État signifie aussi profession, condition. État de mariage. État ecclésiastique. Remplir les devoirs de son état. Ne point sortir de son état. État : la condition de la personne, en tant qu'elle est légitime ou bâtarde, noble ou roturière. On lui dispute son état, on dit qu'il n'est pas légitime, qu'il n'est pas noble. Déchoir de son état (...).

Le mot fait partie du vocabulaire philosophique. Lalande, Vocabulaire..., donne pour premier sens station, par opposition à mouvement, et par suite, détermination consistant en une manière d'être momentanée plus ou moins durable, et non en une action ou un devenir. Sous cet aspect, le sens de état s'oppose à celui de nature.

Le mot état fait l'objet d'une note dans l'édition de P. Sellier (Garnier, p. 618), qui en souligne l'origine jansénienne. Voir les titres des parties de l'Augustinus: De statu naturae lapsae, De statu naturae innocentis. La signification sociale du mot peut être liée aux termes de misère et de grandeur, qui représentent des états.

113 Le mot dont le sens parait le plus proche est condition (au sens où l'on parle de la condition humaine, mais aussi de la condition sociale, etc.). Il implique une idée complexe de permanence dans la constitution de l'individu, tel qu'il est fait, sa « nature, faible ou forte, telle qu'elle est» (Sel.486), mais susceptible de changement, voire de transformation radicale selon le changement des circonstances ou de la disposition. L'état de l'homme n'est pas le même avant et après le péché ; l'état des Juifs n'est pas le même avant la venue du Christ (période durant laquelle il est le peuple prophète) et après la crucifixion. Voir coHEN Lionel, «Pascal et le judaïsme », dont la thèse est qu'il y a dans le pensée de Pascal une étroite analogie entre l'état de l'homme en général et l'état du peuple juif, également composés de grandeur et de misère.

114 La signification physique (états solide, liquide, etc.) n'apparaît qu'ultérieurement.

\section{Sel. 43}

Dans la lettre de l'injustice peut venir,

La plaisanterie des aînés qui ont tout. "Mon ami, vous êtes né de ce côté de la montagne, il est donc juste que votre aîné ait tout. »

«Pourquoi me tuez-vous?» 


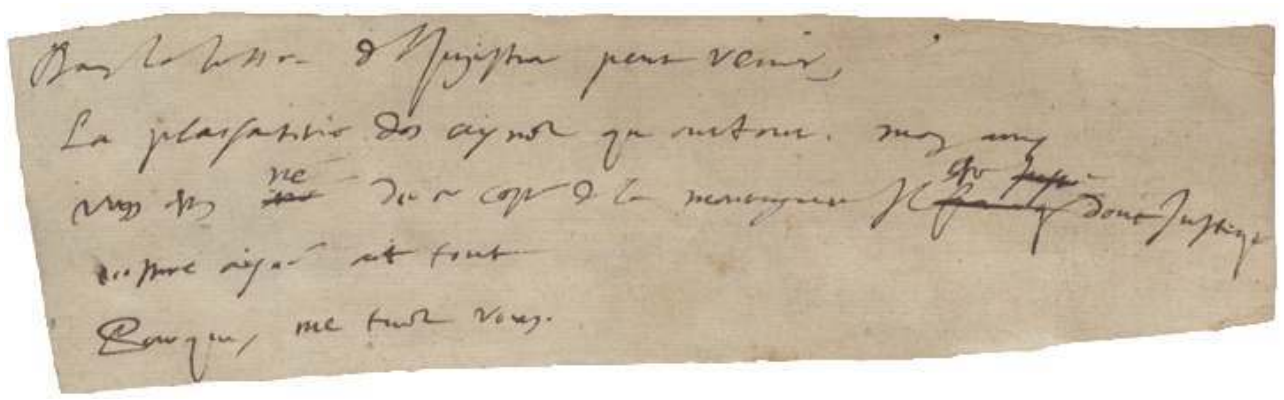

ERNST Pol, Les Pensées de Pascal. Géologie et stratigraphie, p. 232. Strate FNIC.

Crédit Pol Ernst

MESNARD Jean, Les Pensées de Pascal, $2^{e}$ éd., p. 293.

Le mot donc est un ajout.

117 L'expression Mon ami ne doit pas être prise pour une formule d'amitié. C'est au contraire une dénomination au ton hautain, voire méprisant. Voir les fragments Sel. 84.

\section{Fragments connexes}

- Sel. 39 et 41. «Lettre qu'on doit chercher Dieu » renvoie à la «lettre d'exhortation à un ami » de Sel. 39 ; « la machine » du fragment 41 se rapporte au « discours de la machine » de Sel. 45.

- Sel 54.

- Sel. 84.

- Sel. 94, notamment pour le mot plaisanterie.

- Sel. 100 et 101.

- Sel. 108.

\section{Problèmes d'interprétation}

118 Il ne semble pas y avoir d'autre indication sur le projet d'une lettre de l'injustice. Les fragments de la liasse Misère insistent plutôt sur le fait que la véritable justice n'est ni connue ni assignable, que sur l'injustice proprement dite.

119 La question de la nature du génitif dans l'expression la plaisanterie des aînés qui ont tout a été posée : génitif objectif ou subjectif?

L'édition GEF, XIII, p. 213, propose en note une interprétation: quoique rien n'indique «par quel lien ces lettres (sc. que Pascal projetait d'écrire sur l'injustice) se rattachaient à l'ensemble du sujet ", «il est possible que Pascal y visait l'objection que le libertin tire naturellement contre la doctrine janséniste des principes de la justice humaine, à savoir que la récompense des élus et le châtiment des damnés sont également iniques, puisque la grâce a été arbitrairement donnée aux uns et refusée aux autres. Or Pascal nie que la justice humaine puisse juger la justice divine, car la justice humaine est impuissante à se justifier (...). Alors les mystères de la prédestination et de la grâce n'ont plus rien de choquant pour nous, puisque nous avons renoncé à mesurer les choses divines avec nos moyens humains de comprendre ». 
Un rapprochement est à faire avec le $\mathrm{XI}^{\mathrm{e}}$ Provinciale, qui lie le rire et la plaisanterie à la notion de disproportion, qui répond bien à ce fragment.

\section{La plaisanterie des aînés qui ont tout}

Sur la disposition juridique du droit d'aînesse, voir :

- PILloRget René et Suzanne, France baroque France classique, II, Dictionnaire, p. 30-31. Cette disposition du droit féodal a pour but originel d'assurer le service du fief au seigneur dont il relevait. Par la suite, il garantit à l'aîné, chargé de perpétuer le lignage, une part important des héritages. Certaines coutumes comme celle de Bretagne, vont très loin dans ce sens. Le droit d'aînesse est un droit absolu : les pères et mères ne peuvent disposer de leurs fiefs en faveur des puînés, ni ordonner qu'il en soit fait un partage égal. Pour ce qui concerne les successions roturières, le droit d'aînesse n'existe pas, et il y a prédominance du partage égal.

- DOMAT Jean, Lois civiles, p. LVI sq. Les lois arbitraires, celles qu'une autorité légitime peut établir, changer, abolir selon le besoin, et peuvent être changées sans violer l'esprit des lois fondamentales, sont nécessaires pour régler certaines difficultés nées de l'application des lois immuables : par exemple pour les problèmes relatifs à l'héritage (p. LVIII).

- DOMAT, Les lois civiles dans leur ordre naturel, Seconde partie. Des successions, p. 301 sq. Voir les sections :

I. Pourquoi on a distingué les successions des engagements.

II. Nécessité des successions, et comment elles ont été réglées par les lois, p. 301.

III. Cas des successions qu'on appelle légitimes, ou testamentaires.

IV. Ordres des successions légitimes.

VIII. L'ordre par intestat dépend de la volonté de Dieu ; l'ordre par testament dépend de la volonté des hommes : p. 305.

Seconde partie. Des successions, p. 355 sq. Livre second. Des successions légitimes ou ab intestat. Section II, p. 357.

Pour envisager le problème sous l'angle biographique, voir $O C$ II, p. 864 sq. On peut aussi se rapporter à la situation des Roannez. Voir MESNARD Jean, Pascal et les Roannez, I, p. 108.

\section{Sel. 44}

« Les misères de la vie humaine ont frondé tout cela.

Comme ils ont vu cela ils ont pris le divertissement. » 

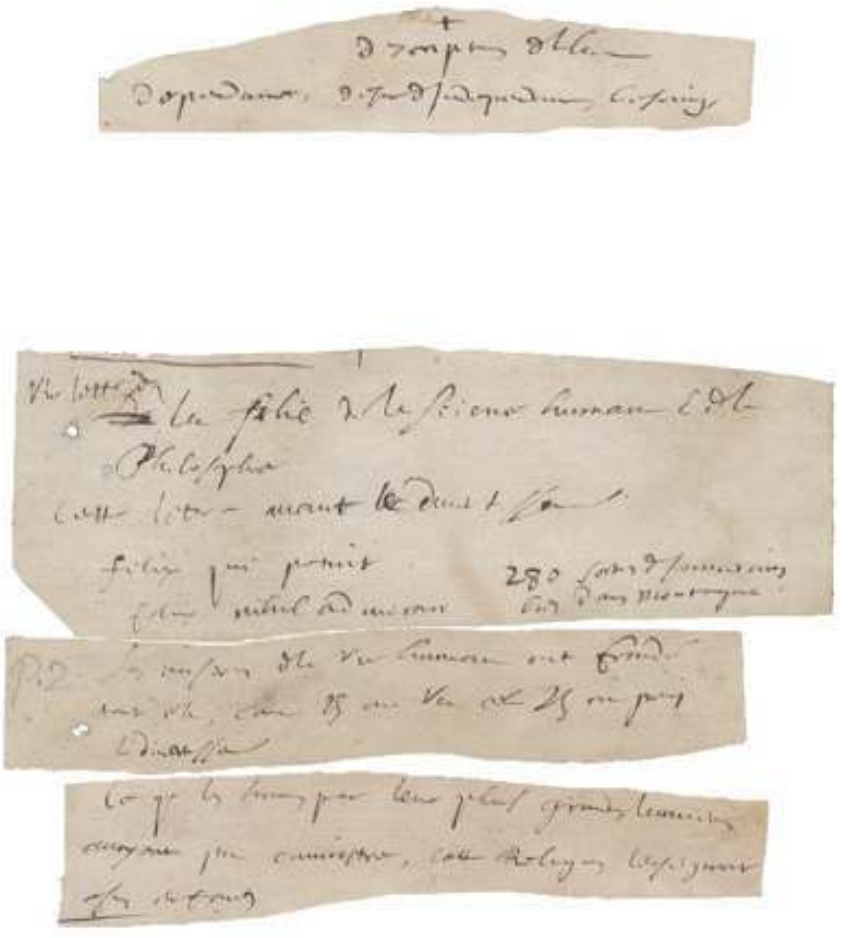

ERNST Pol, Géologie..., Album I, p. 23. Cette reconstitution ne figure pas dans l'édition de l'ouvrage de Pol Ernst par la Voltaire Foundation). Elle est hypothétique pour une moitié de feuillet de format présumé 20 × 30, non identifié :

Ennui, Laf. 78, Sel. 113 (hypothétique).

I, Laf. 408, Sel. 27.

Ordre, Laf. 10, Sel. 44.

Fondements, Laf. 229, Sel. 261 (hypothétique).

Le manuscrit n'est pas raturé. II n'y a qu'un état du fragment.

Crédit Pol Ernst

Suematsu Hisashi, «Les Pensées et le métatexte », Équinoxe, 1, automne 1987, p. 27-53. Voir p. 45.

Le mot cela, employé deux fois, pose un problème d'interprétation. S'agit-il du désaccord sur la nature du souverain bien?

Le mot misères est bien au pluriel. Voir Sel. 33 : nos misères, la plus grande de nos misères.

\section{Fronder / fonder}

Dictionnaire de l'Académie, Fronder : jeter, ruer des pierres avec une fronde ; il se dit aussi de tout ce qu'on jette avec violence. Il signifie figurément blâmer, condamner, critiquer hautement. Il n'eut pas sitôt ouvert la bouche, que tout le monde le fronda. On a frondé sa harangue. Fronder signifie aussi parler contre le gouvernement. C'est un homme qui ne fait que fronder tout le jour. Dans ce sens il est neutre. Fronder est attesté par Richelet (Le Guern).

130 M. Le Guern a le premier noté la lecture frondé, que donne aussi l'édition Sellier. L'édition Martineau lit fondé; elle associe ce fragment avec Sel. 690, c'est-à-dire à un texte où Pascal se moque de la tranquillité des hommes. 
Voir la note de l'édition Sellier-Ferreyrolles, p. 56. Il faut comprendre : ces misères ont amené les hommes à contester la prétention des philosophes à leur faire attendre le souverain bien. C'est pourquoi Pascal avait prévu de faire suivre la Lettre de la folie de la science humaine et de la philosophie par le chapitre Divertissement.

\section{Sel. 45}

Ordre. Après la lettre qu'on doit chercher Dieu, faire la lettre d'ôter les obstacles, qui est le discours de la machine, de préparer la machine, de chercher par raison.

Sel.45

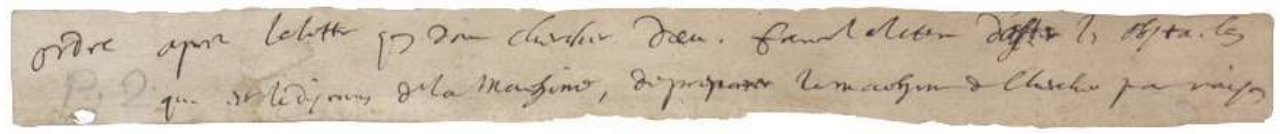

ERNST Pol, Les Pensées de Pascal. Géologie et stratigraphie, p. 232

Crédit Pol Ernst

ERNST Pol, Les Pensées de Pascal. Géologie et stratigraphie, p. 232. Strate FNIC, c'est-à-dire datant du printemps 1658. Cela va dans le sens de l'hypothèse de P. Sellier sur le sens de la liasse Ordre.

Sur le manuscrit, le mot Ordre est en marge de gauche, à part du reste. Le verbe ôter est raturé.

Sur la Machine, voir Sel. 681. Voir in GEF XIII, p. 180, la note de Brunschvicg : préparer la machine, c'est prendre l'attitude et pratiquer les œuvres du chrétien, c'est quitter les plaisirs et chasser les passions.

\section{Indications sur l'ordre}

SELLIER Philippe, Port-Royal et la littérature, I, Pascal, p. 55 sq. La lettre pour porter à chercher Dieu. La lettre d'ôter les obstacles: p. 59 sq. Voir p. 66, n. 22 : une lettre ou quatre lettres? Sellier préfère la seconde hypothèse, quatre lettres qui correspondent «à la succession des quatre discours de l'ouverture ".

Noter que l'expression «faire la lettre » suppose qu'elle n'est pas encore composée. Il s'agirait du projet d'un texte sur la suppression des obstacles qui prendra la forme d'une lettre.

\section{Ôter les obstacles}

- Voir in GEF XIII, p. 180, la note de Brunschvicg : les obstacles, c'est « le double libertinage de la vie et de la pensée ». Préparer la machine, c'est prendre l'attitude et pratiquer les œuvres du chrétien, c'est quitter les plaisirs et les passions.

- Voir Sel. 680 ; et Sel. 699, sur l'amour-propre et la concupiscence, obstacles qui arrêtent l'homme et l'empêchent d'aimer Dieu.

- Le fragment 168, Divertissement, indique les obstacles extérieurs. 
- Voir Méthodes chez Pascal, p. 257 et 264.

\section{De chercher par raison}

Voir in GEF XIII, p. 180, la note de Brunschvicg : chercher par raison, «c'est se guérir du scepticisme en comprenant la relation que la raison soutient avec la foi ».

Problème d'interprétation : le Discours de la machine (l'argument du pari) ne démontre pas qu'on doit chercher par raison, c'est-à-dire par le moyen de la raison; c'est même le contraire. En fait, il faut comprendre que c'est par la raison qu'on montre qu'il faut cherche $\mathrm{r}$ : il est raisonnable de chercher; et il faut continuer parce qu'on n'a pas trouvé. Cela répond bien à Infini rien.

\section{Sel. 46}

Ordre.

Les hommes ont mépris pour la religion, ils en ont haine et peur qu'elle soit vraie. Pour guérir cela il faut commencer par montrer que la religion n'est point contraire à la raison. Vénérable, en donner respect. La rendre ensuite aimable, faire souhaiter aux bons qu'elle fût vraie et puis montrer qu'elle est vraie.

Vénérable parce qu'elle a bien connu l'homme.

Aimable parce qu'elle promet le vrai bien.

Sel.46

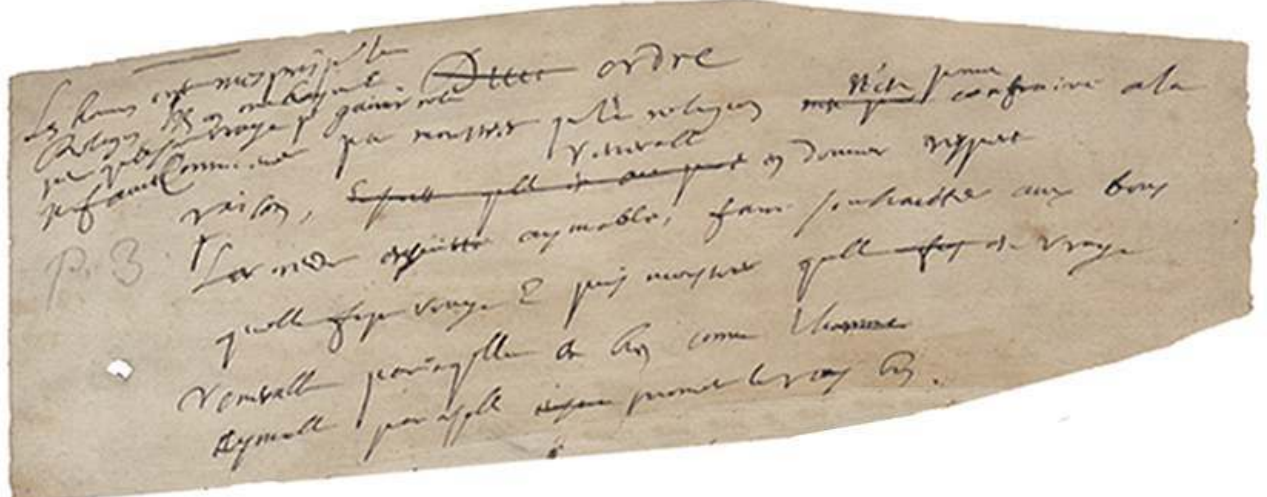

ERNST Pol Géologie..., p. 290. Petit feuillet, hapax non identifié. II semble plus ancien que les autres.

Cliché Pol Ernst

\section{Texte initial (d'après la restitution de Y. Maeda)}

n'est point

Commencer par montrer que la religion n'est point contraire à la

Vénérable

raison. Ensuite qu'elle a pre en donner respect.

La rendre ensuite aimable, faire souhaiter aux bons

qu'elle fût vraie et puis montrer qu'elle est vraie. 
Vénérable parce qu'elle a bien connu l'homme.

Aimable parce qu'elle ensei promet le vrai bien.

- ERNST Pol Géologie..., p. 290. Petit feuillet, hapax non identifié. Il semble plus ancien que les autres.

- MAEDA Yoichi, Commentaire sur les Pensées, I, p. 55. Les premières lignes ont été surajoutées. Pascal a commencé par jeter sur le papier son projet d'ordre ; il a ensuite expliqué à quelle disposition d'esprit cela correspond.

\section{Le titre}

141 Le titre pose un problème: on lit Divi, peut-être pour Division. Ordre se serait donc substitué très tôt à division. Voir sur le rapport de ces deux termes le fragment Sel. 562. Voir le fragment Sel. 226, où l'on voit aussi qu'un ajout fait apparaître un concept clé dans le titre (la prévention).

\section{Interprétation du fragment}

- suematsu Hisashi, «Les Pensées et le métatexte », Équinoxe, 1, automne 1987, p. 27-53. En dehors de la première phrase, ce texte est entièrement un métatexte : p. 46. Un corps de texte discursif est annoncé ou résumé par un fragment (métatexte) qui est à son tour dénommé par un titre (métatexte au second degré) : p. 46. Autres fragments de même structure dans la liasse Ordre: Laf. 4, 5, 8 et 11.

Ce fragment semble en désaccord avec Sel. 40. En revanche, il se rapporte aisément à l'ordre des liasses. Ce dernier texte de la liasse est aussi le plus récent ; il est probable que Sel. 40 n'est qu'une étape intermédiaire.

- MESNARD Jean, Les Pensées de Pascal, p. 179. Ce programme ne s'applique en toute rigueur qu'à la seconde partie de l'Apologie, où l'exposé des preuves proprement dites (ch. 18 à 25) est précédé d'un ensemble de chapitres (11 à 17) insistant sur le caractère raisonnable de la religion. Cet ensemble doit être précédé par une définition de l'homme.

- LE GUERN : ce fragment annonce du thème de la liasse Soumission et usage de la raison.

- Paolo SERINI renvoie à la XI ${ }^{\mathrm{e}}$ Provinciale: «Il y a deux choses dans les vérités de notre religion: une beauté divine qui les rend aimables, et une sainte majesté qui les rend vénérables ».

- SELLIER Philippe, Port-Royal et la littérature. I, p. 65

- SAINTE-BEUVE, Correspondance littéraire, provenant pour la plus grande partie du fonds Lebrun de la Bibliothèque Mazarine, avec introduction et notes de Guy de la Batut, éd. Montaigne, Paris, 1929, 158 p., lettre à Faugère datée d'un vendredi (1869 ?), dans laquelle Sainte-Beuve lui demande s'il reproduit et ponctue correctement le fragment.

- Gouhier Henri, Blaise Pascal. Conversion et apologétique, p. 103 sq. Analyse de ce fragment. Rapport avec le fragment Infini rien, pour l'idée de rendre la religion aimable.

- Pensées, éd. Havet, II, p. 100-101, et commentaire p. 134-135.

\section{Les hommes ont mépris pour la religion}

- DROZ Édouard, Étude sur le scepticisme de Pascal considéré dans le livre des Pensées, p. 55 sq.

- HAVET, édition des Pensées, II, p. 134. «Où Pascal est-il emporté par son humeur? S'il était vrai que la religion, telle qu'il la présente, n'inspirât aux hommes que du mépris, de la haine 
et de l'effroi, serait-ce la condamnation de la nature humaine, ou celle d'une foi farouche et bizarre, foi de sectaire et de malade ? Port-Royal supprime ces paroles si dures ».

- Voir Sel. 743, sur la haine de la vérité.

\section{Peur qu'elle soit vraie} donne pas de richesses".

Source : Charron, Les trois vérités, II, 10. venu, non pour une seule nation, mais pour tous.

Voir les fragments

- Sel. 6 .

- Sel. 248, note de conclusion.

- Sel. 249.

- Sel. 681.

- Voir Sel. 493 : «Jésus-Christ aimable parce qu'il n'a pas dompté les païens par la force, et ne

- Voir Sel. 389 : la religion chrétienne rend l'homme aimable.

Le fragment donne un ordre de priorités, répondant à l'Art de persuader. Les hommes prétendent ne suivre que le vrai, et en réalité ils ne suivent que ce qu'ils aiment; Pascal rejette donc à la fin de la démonstration la preuve de la vérité du christianisme.

Rendre la religion aimable aux bons : aimable parce qu'elle connaît le vrai bien. C'est l'objet de la liasse XVII Rendre la religion aimable, qui n'a que deux fragments. Ces deux fragments vont dans deux sens différents: Sel. 254 indique «J.-C. pour tous »; Sel. 255 indique «il n'y a point de rédempteur pour les païens». Voir ERNST Pol, Approches pascaliennes, p. 312 sq. Dans le cas présent, l'argument reposait sur le fait que Jésus est

MESNARD Jean, Les Pensées de Pascal, 2e éd., p. 176 et 187.

\section{Faire souhaiter aux bons qu'elle fût vraie}


HAVET, édition des Pensées, II, p. 134. Port-Royal supprime aux bons. Havet interprète cette suppression par le fait que c'est une tautologie, le souhait que la religion soit vraie n'appartient qu'aux bons, ne pouvant être inspiré que par la grâce.

\section{BIBLIOGRAPHIE}

\section{L'apologétique de Pascal}

ASTIE J.F., «L'apologie récusée par le Vicaire savoyard et l'apologie irrécusable de Pascal », Revue chrétienne, 1954, p. 347-359 et 405-417.

BAUDIN Émile, La Philosophie de Pascal, La Baconnière, Neuchâtel, 1946-1947, 4 vol.

BENDA Julien, « Pascal et le libertin », Revue hebdomadaire, Numéro du centenaire de Pascal, Paris, 1923.

BERGER C., Essai sur l'apologétique de Pascal, Forestié, Montauban, 1869, 30 p.

BOUCHILLOUX Hélène, « La critique des preuves de l'existence de Dieu et la valeur du discours apologétique ", in COMTE-SPONVILLE André (dir.), Pascal philosophe, Revue Internationale de Philosophie, nº 1, 1997, p. 5-30.

BOUCHILLOUX Hélène, Apologétique et raison dans les Pensées de Pascal, Paris, Klincksieck, 1995.

BUSSON Henri, La pensée religieuse française de Charron à Pascal, Vrin, Paris, 1933, 622 p.

CHAVANNES F., « Des Pensées de Pascal et de l'apologétique chrétienne », Le Lien, 29 janv., p. 17-19, et 12 fév. 1859, p. 25-26.

CHAUCHARD Paul, « Deux maîtres de l'apologétique chrétienne : Pascal et Teilhard de Chardin », Tricentenaire de la mort de Pascal, La Table ronde, 171, avril 1962, p. 97-103.

DEDIEU J., « Survivances et influences de l'apologétique traditionnelle dans les Pensées », Revue d'Histoire Littéraire de la France, 1930-1931, Colin, Paris ; 1930 : p. 481-513 ; 1931 : p. 1-39.

DUMAS André, «L'apologétique du Dieu caché chez Pascal », Revue d'histoire et de philosophie, $\mathrm{n}^{\circ} 4$, 1962, p. 290-303.

EMPART L., «La valeur apologétique des Pensées de Pascal », Annales de philosophie chrétienne, juin 1880, p. 238-248.

ERNST Pol, La trajectoire pascalienne de l'Apologie. Étude critique comparée de la lissa A.P.R., du Discours de Filleau et de la Préface d'Etienne Périer, Archives des Lettres Modernes, 84, Paris, Minard, 1967.

FORCE Pierre, «Conditions d'efficacité du discours apologétique dans les Pensées », in FERREYROLLES Gérard (dir.), Littérature et religion, Littératures classiques, $\mathrm{n}^{\circ} 39$, Printemps $2000, \mathrm{p}$. 197-206.

GORY A., Des Pensées de Pascal considérées comme apologie du christianisme et des conditions actuelles de l'apologétique, Guy, Laigle, 1883, 102 p.

GOUHIER Henri, Blaise Pascal. Conversion et apologétique, Vrin, Paris, 1986, 268 p. 
HUBERT Marie Louise, Pascal's Unfinished Apology : a study of his plan, Paris, Presses Universitaires de France, 1952.

JOURNET Ch., Vérité de Pascal. Essai sur la valeur apologétique des Pensées, Saint Maurice, 1951, 325 p.

JULIEN EYMARD D’ANGERS, Pascal et ses précurseurs. L'apologétique en France de 1580 à 1670, Nouvelles Éditions Latines, Paris, 1954, 243 p.

JULIEN EYMARD D'ANGERS, « Les Pensées de Pascal à la lumière des apologétiques de son temps ", Pascal, Textes du tricentenaire, Société des Amis de Port-Royal, n 11-14, 1963.

KAUFMANN M., « Pascal's apologia », The Quarterly review, n 406, janv. 1906, p. 526-552.

KOLAKOWSKI Leszek, Dieu ne nous doit rien. Brève remarque sur la religion de Pascal et l'esprit du jansénisme, Albin Michel, Paris, 1997, 318 p.

LABERTHONNIERE, «L'apologétique et la méthode de Pascal », Revue du Clergé Français, Lethielleux, 1903 ; repris in Essais de philosophie religieuse, Lethielleux, 1903.

LABRIERE Yves de, «L'apologétique de Pascal et la mort de Pascal », Études, 1911, 129, p. 635-636.

LACOMBE R.E., L'apologétique de Pascal. Étude critique, Presses Universitaires de France, Paris, 1958, $320 \mathrm{p}$.

LONGHAYE G., « Pascal apologiste, valeur utile des Pensées », Études religieuses, philosophique, historiques et littéraires, 15 décembre 1891, p. 521-542.

MARITAIN Jacques, «Pascal apologiste », Troisième centenaire de Pascal, La Revue hebdomadaire, $\mathrm{n}^{\circ}$ 28, 14 juillet 1923, p. 184-200.

MASSAUT J. P., « Thomisme et augustinisme dans l'apologétique du XVII ${ }^{\mathrm{e}}$ siècle », Revue des sciences philosophiques et théologiques, t. XLIV, p. 617-638.

MAUDUIT Michel, Traité de religion contre les athées, les déistes et les nouveaux pyrrhoniens, éd. HYUN Mi-ae, Faculté des Lettres et Sciences Humaines de l'Université Blaise-Pascal, Clermont-Ferrand, 1996, 400 p.

MESNARD Jean, Pascal, Les écrivains devant Dieu, Desclée de Brouwer, Paris, 1965, 142 p.

MESNARD Jean, « Au cœur de l'apologétique pascalienne : Dieu par Jésus-Christ », in La culture du XVII siècle, Paris, P.U.F., 1992, p. 414-425.

MONOD A., De Pascal à Chateaubriand. Les défenseurs du christianisme de 1670 à 1802. Alcan, Paris, 608 p.

NAVILLE E., «L'apologie de Pascal a-t-elle vieilli ? », Bibliothèque universelle, Revue suisse et étrangère, juillet 1858, p. 384-412.

NOURRY, «Le rôle des miracles dans l'apologétique pascalienne », Annales de philosophie chrétienne , avril 1898.

OLIVO Gilles, « Pascal apologiste et philosophe : bref état de la question », XVII ${ }^{e}$ siècle, oct.-déc. 1994, n 185, p. 731-738.

PUGH Anthony R., The composition of Pascal's Apologia, Toronto, University of Toronto Press, 1984. RECOLIN Numa, L'apologétique de Pascal dans le livre des Pensées, Thèse, Toulouse, 1850.

RENNES J., Pascal et le libertin, Librairie Valois, Paris, 1950, 242 p.

RUSSIER Jeanne, La foi selon Pascal, I, Dieu sensible au cœur, II, Tradition et originalité dans la théologie pascalienne de la foi, Paris, Presses Universitaires de France, 1949, 2 vol. 
SELLIER Philippe, «Le « fondement » prophétique dans les Pensées », Courrier du Centre International Blaise Pascal, 16, 1994, p. 21-24.

SULLY-PRUDHOMME, « La méthode de Pascal apologiste chrétien », Revue de Paris, $1^{\mathrm{er}}$ septembre 1894, p. 5-22.

THOMAS J.F., Les caractères de la démonstration dans l'Apologie pascalienne, Nizet et Bastard, Paris, 1942, $176 \mathrm{p}$.

VINET A., Études sur Blaise Pascal, p. 13 sq.

INDEX

Mots-clés : Pascal, Pensées

\section{AUTEUR}

DOMINIQUE DESCOTES

CERHAC, Université Blaise Pascal 